\title{
Altered Electrical, Biomolecular, and Immunologic Phenotypes in a Novel Patient-Derived Stem Cell Model of Desmoglein-2 Mutant ARVC
}

\author{
Robert N. Hawthorne ${ }^{1,2} \mathbb{D}^{D}$, Adriana Blazeski ${ }^{1}$, Justin Lowenthal ${ }^{1,2} \mathbb{D}$, Suraj Kannan ${ }^{1,2}$, Roald Teuben ${ }^{1}$, \\ Deborah DiSilvestre $^{3}$, Justin Morrissette-McAlmon ${ }^{1}$, Jeffrey E. Saffitz ${ }^{4}$, Kenneth R. Boheler ${ }^{1,3}$, \\ Cynthia A. James ${ }^{3}$ (D), Stephen P. Chelko ${ }^{3,5, *(\mathbb{D})}$, Gordon Tomaselli ${ }^{3,6, *}$ and Leslie Tung ${ }^{1, *}$
}

check for

updates

Citation: Hawthorne, R.N.; Blazeski, A.; Lowenthal, J.; Kannan, S.; Teuben, R.; DiSilvestre, D.; MorrissetteMcAlmon, J.; Saffitz, J.E.; Boheler, K.R.; James, C.A.; et al. Altered Electrical, Biomolecular, and Immunologic Phenotypes in a Novel Patient-Derived Stem Cell Model of Desmoglein-2 Mutant ARVC. J. Clin. Med. 2021, 10, 3061. https://doi.org/ $10.3390 /$ jcm10143061

Academic Editor: Gani Bajraktari

Received: 29 May 2021

Accepted: 8 July 2021

Published: 10 July 2021

Publisher's Note: MDPI stays neutral with regard to jurisdictional claims in published maps and institutional affiliations.

Copyright: (C) 2021 by the authors Licensee MDPI, Basel, Switzerland. This article is an open access article distributed under the terms and conditions of the Creative Commons Attribution (CC BY) license (https:/ / creativecommons.org/licenses/by/ $4.0 /)$.
1 Department of Biomedical Engineering, School of Medicine, Johns Hopkins University, Baltimore, MD 21205, USA; rhawtho4@jhmi.edu (R.N.H.); ablazesk@mit.edu (A.B.); jilowenthal@jhmi.edu (J.L.); skannan4@jhmi.edu (S.K.); rteuben1@jhmi.edu (R.T.); jmorri65@jhmi.edu (J.M.-M.); kbohele1@jhmi.edu (K.R.B.)

2 Medical Scientist Training Program, School of Medicine, Johns Hopkins University, Baltimore, MD 21205, USA

3 Department of Medicine, School of Medicine, Johns Hopkins University, Baltimore, MD 21205, USA; ddisilvestre@som.umaryland.edu (D.D.); cjames7@jhmi.edu (C.A.J.)

4 Department of Pathology, Beth Israel Deaconess Medical Center, Boston, MA 02215, USA; jsaffitz@bidmc.harvard.edu

5 Department of Biomedical Sciences, College of Medicine, Florida State University, Tallahassee, FL 32306, USA

6 Department of Medicine, Albert Einstein College of Medicine, Bronx, NY 10461, USA

* Correspondence: stephen.chelko@med.fsu.edu (S.P.C.); gordon.tomaselli@einsteinmed.org (G.T.); ltung@jhu.edu (L.T.); Tel.: +1-850-644-2215 (S.P.C.); +1-718-430-2801 (G.T.); +1-410-955-9603 (L.T.)

\begin{abstract}
Arrhythmogenic right ventricular cardiomyopathy (ARVC) is a progressive heart condition which causes fibro-fatty myocardial scarring, ventricular arrhythmias, and sudden cardiac death. Most cases of ARVC can be linked to pathogenic mutations in the cardiac desmosome, but the pathophysiology is not well understood, particularly in early phases when arrhythmias can develop prior to structural changes. Here, we created a novel human induced pluripotent stem cell-derived cardiomyocyte (hiPSC-CM) model of ARVC from a patient with a c.2358delA variant in desmoglein-2 (DSG2). These DSG2-mutant (DSG2 ${ }^{\mathrm{Mut}}$ ) hiPSC-CMs were compared against two wildtype hiPSCCM lines via immunostaining, RT-qPCR, Western blot, RNA-Seq, cytokine expression and optical mapping. Mutant cells expressed reduced DSG2 mRNA and had altered localization of desmoglein-2 protein alongside thinner, more disorganized myofibrils. No major changes in other desmosomal proteins were noted. There was increased pro-inflammatory cytokine expression that may be linked to canonical and non-canonical NFKB signaling. Action potentials in DSG2 ${ }^{\text {Mut }} \mathrm{CMs}$ were shorter with increased upstroke heterogeneity, while time-to-peak calcium and calcium decay rate were reduced. These were accompanied by changes in ion channel and calcium handling gene expression. Lastly, suppressing DSG2 in control lines via siRNA allowed partial recapitulation of electrical anomalies noted in DSG2 ${ }^{\text {Mut }}$ cells. In conclusion, the aberrant cytoskeletal organization, cytokine expression, and electrophysiology found DSG2 ${ }^{\text {Mut }}$ hiPSC-CMs could underlie early mechanisms of disease manifestation in ARVC patients.
\end{abstract}

Keywords: arrhythmogenic cardiomyopathy; arrhythmogenic right ventricular cardiomyopathy; ARVC; desmoglein-2; NFKB signaling; patient-derived stem cells; induced pluripotent stem cells

\section{Introduction}

Arrhythmogenic right ventricular cardiomyopathy (ARVC) is an inherited, progressive arrhythmogenic cardiomyopathy (ACM) characterized by cardiomyocyte death and fibrofatty scarring in the ventricular myocardium. Clinically, patients with ARVC develop ventricular arrhythmias which often present more frequently as the disease progresses. 
ARVC is estimated to account for more than $10 \%$ of all cardiovascular deaths in patients younger than 65 years old and is a leading cause of sudden cardiac death (SCD) in young athletes [1-3]. ARVC prevalence is approximately 1:1000-1:5000, and patients are typically diagnosed in young adulthood; diagnosis before puberty is extremely rare [4-6].

While the pathophysiology underlying ARVC is still poorly understood, there has been general scientific consensus that the common final pathway of the disease involves the disruption of the cardiac desmosome, a cellular structure present at the intercalated disc that is critical for intercellular mechanical and electrical coupling between cardiomyocytes $[7,8]$. Pathogenic variants in any of the five cardiac desmosomal genes - plakoglobin (JUP), plakophilin-2 (PKP2), desmoglein-2 (DSG2), desmoplakin (DSP) and desmocollin-2 (DSC2) - account for more than $60 \%$ of inherited ARVC and have a combined estimated prevalence of approximately 1.2 per 1000 in the general population [9]. Among these, PKP2 and DSG2 are the first and second most common variants, respectively $[6,10]$.

Determining the pathogenic mechanisms underlying ARVC has proven difficult. The study of human tissue is challenging, as ARVC is frequently diagnosed in later stages of disease (e.g., myocardial scarring) when retrieval of a myocardial biopsy presents significant risk of cardiac perforation. And while patients with advanced ARVC develop severe myocardial scarring and cardiomyocyte loss, which can contribute to arrhythmic risk, substantial evidence demonstrates that cardiac electrical abnormalities and elevated risk of dangerous arrhythmias-develop well before any overt structural deterioration can be detected [11-13]. Several animal models of ARVC have been established, including in mice [14-17] and dogs [18,19], which have proven useful in studying specific pathophysiologic processes, but these models are limited due to substantial differences in cardiac electrophysiology between animals and humans [20]. Human induced pluripotent stem cell-derived cardiomyocytes (hiPSC-CMs) present a powerful model for studying cardiac disease, and ARVC patient-specific hiPSC-CMs have been shown to recapitulate key characteristics observed in human disease [21]. By generating and studying hiPSC-CMs harboring unique pathogenic variants associated with ARVC, we can begin to uncover the mechanisms by which diseased cardiomyocytes behave differently in this early "concealed phase" of ARVC, revealing new potential targets for therapeutic intervention.

Prior studies show that PKP2-mutant hiPSC-CMs display reduced membrane localization of plakophilin-2 and plakoglobin, in conjunction with disrupted desmosomal structure [22,23]. These models have also revealed a number of electrical defects. PKP2-mutant hiPSC-CM models display altered calcium homeostasis and connexin-43 function [17] while both PKP2- and DSG2-mutant hiPSC-CM models demonstrated slower action potential upstrokes and reduced sodium current density [24,25]. Inflammatory infiltration by immune cells has been shown to play an important role in pathologic remodeling of the myocardium in ARVC, and recent studies have indicated that ARVC hiPSC-CMs produce and secrete inflammatory cytokines via innate activation of nuclear factor- $\mathrm{KB}$ (NFKB) [15]. In Dsg2-mutant mice, inhibition of NFKB via the small molecule antagonist, Bay11-7082, prevented myocardial NFKB-mediated cytokine storm and inflammatory infiltration.

In this study, we developed a novel hiPSC-CM model of ARVC derived from a patient with a likely pathogenic variant in DSG2. We characterized a variety of functional and molecular features that could help link the cellular behavior with clinical manifestation of ARVC. Our findings show that hiPSC-CMs harboring this mutation display altered electrophysiological properties and augmented immune cytokine expression, even when compared to ARVC hiPSC-CMs reported in other studies, suggesting that significant phenotypic variation exists among models with different causative variants.

\section{Materials and Methods}

\section{1. hiPSC Line Generation}

Blood samples were collected from the donor (JHU013), who met 'definite' 2010 Task Force Criteria for diagnosis of ARVC [26]. Peripheral blood mononuclear cells (PBMCs) were isolated and reprogrammed into pluripotency via Sendai-viral transfection of the 
Yamanaka factors (see Supplementary Methods for complete protocol). Clonal colonies were selected and expanded until passage 10. DNA was then isolated to verify the loss of the Sendai virus and clones were screened via Sanger sequencing and karyotyping.

\section{2. hiPSC-CM Differentiation and Culture}

In addition to the DSG2 c.2358delA hiPSC line generated, we used two control lines. One was generated from an age- and sex-matched donor under identical protocols (JHU001, "Ctrl1") $[27,28]$ and the other is a widely used wildtype control line kindly gifted to us by Dr. Bruce Conklin at the University of California, San Francisco (WTC11, "Ctrl2") [29], which can be obtained from the NIGMS Human Genetic Cell Repository at the Coriell Institute for Medical Research: GM25256.

For the detailed differentiation protocol please refer to Supplementary Methods. Briefly, hiPSCs were subjected to cardiogenic differentiation via previously described methods using small molecule modulation of Wnt signaling [30,31]. Spontaneous beating was observed around day 7-9. Beginning between days 10-14, hiPSC-CM cultures underwent 4-day purification in glucose-depleted DMEM supplemented with $4 \mathrm{mM} \mathrm{L}$-lactate (lactate medium; MilliporeSigma, St. Louis, MO, USA). On differentiation day 28, hiPSC-CMs were re-plated in RPMI1640 plus B27 supplement (B27+ media; Thermo Fisher, Waltham, MA, USA) onto Geltrex-coated Thermanox coverslips (Thermo Fisher) at a density of 250,000 cells $/ \mathrm{cm}^{2}$. hiPSC-CM monolayers were then cultured for $7-10$ days, with media changes every other day, until evaluation.

\subsection{Biomolecular Characterization Using Immunostaining, Real-Time Quantitative PCR, Western Blot, and Cytokine Arrays}

For immunostaining, cells were fixed in $4 \%$ paraformaldehyde, and probed using standard immunocytochemistry techniques. hiPSCs were stained for OCT3/4, SOX2, and Nanog, and hiPSC-CM monolayers were stained for desmoglein-2, plakoglobin, plakophilin-2, cardiac troponin I, $\alpha$-actinin and nuclei (see Table S3 for primary antibody details). Images were acquired via confocal microscopy (ZEISS, Oberkochen, Germany). To assess Z-line length in hiPSC-CMs, $20 \mu \mathrm{m} \times 20 \mu \mathrm{m}$ regions of interest were selected from high magnification images at random by computer script, and $\alpha$-actinin-immunopositive lines were measured by a blinded observer using image analysis software (ImageJ, Version 2.0.0-rc-69/1.53c).

For RT-qPCR experiments, total RNA was extracted from hiPSC-CMs on differentiation day 37-39 using a column preparation (RNeasy Mini Kit; Qiagen Sciences, Germantown, MD, USA) and reverse transcription was performed using the High-Capacity cDNA Reverse Transcription Kit (Applied Biosystems, Waltham, MA, USA). cDNA was amplified using PowerUp SYBR Green PCR master mix (Applied Biosystems) and a QuantStudio 7 Flex RT-qPCR system (Applied Biosystems) under manufacturer's recommended protocol. All assays were performed with three technical replicates and a minimum of three pooled cell samples. GAPDH and 18S rRNA were used in combination as internal controls; all primer sequences are listed in Table S1.

For Western blots, total protein was extracted from hiPSC-CMs using RIPA lysis buffer (Cell Signaling, Danvers, MA, USA). Samples were prepared and run under denaturing conditions on NuPAGE 4-12\% Bis-Tris gels in MOPS buffer using manufacturers recommended reagents and protocol (Thermo Fisher). Proteins were transferred to PVDF membranes using the Trans-Blot Turbo system (Bio-Rad Laboratories, Hercules, CA, USA), probed with primary antibodies (see Table S3) and visualized with fluorescent secondary antibodies (LI-COR, Lincoln, NE, USA). Images were obtained and analyzed using an Odyssey CLx imaging system (LI-COR).

For cytokine analysis, supernatant was aspirated from day 37 hiPSC-CMs, two days after feeding and flash frozen until analysis. Then, hiPSC-CMs were isolated and lysed in RIPA buffer as described above. Cell lysates and cell culture supernatants were analyzed using the Proteome Profiler Human XL Cytokine Array Kit (R\&D Systems, Minneapolis, 
MN, USA), and films were scanned and analyzed using Quick Spots image analysis software (Version 25.0.0r, Western Vision Software, Salt Lake City, UT, USA).

\subsection{RNA Sequencing and Analysis}

hiPSC-CMs cultured to differentiation day 35 were gently dislodged with a cell scraper (Thermo Fisher), suspended in TRIzol reagent and flash frozen for storage. Isolated RNA across the samples had a mean RIN of 9.2. RNA-Seq libraries were prepared by an outside vendor (GENEWIZ, South Plainfield, NJ, USA) using a standard poly-A selection protocol for isolating mRNAs. Final libraries were sequenced as $2 \times 150 \mathrm{bp}$ paired-end sequencing on an Illumina HiSeq. Samples were sequenced to a depth of 30-40 million paired reads/cell. Libraries were mapped to the human genome (GRCh38) using STAR (2.3.1a) [32] with two-pass mode, and then counted using featureCounts (2.0.0) through the Subread package (Version 2.0.2) [33,34]. Identification of differentially expressed genes was done using DESeq2 [35], with genes identified as differentially expressed by Benjamini-Hochbergadjusted $p$-value $<0.05$. KEGG pathway maps were made using the Pathview package (Version 1.26.0) [36].

\subsection{Optical Mapping of Calcium and Transmembrane Potential}

All staining and mapping steps were performed in Tyrode's solution (see Supplementary Methods) containing $10 \mu \mathrm{M}$ blebbistatin (PeproTech, Cranbury, NJ, USA) to suppress contraction and prevent motion artifact. Coverslips with hiPSC-CM monolayers were stained with either the voltage indicator di-4-ANEPPS $(10 \mu \mathrm{M}, 10 \mathrm{~min}$; Thermo Fisher) or calcium indicator Rhod-2 AM ( $1 \mu \mathrm{M}, 15 \mathrm{~min}$; Thermo Fisher); to aid cell loading, staining solutions were supplemented with $0.05 \%$ Pluronic F-127. Coverslips were then gently washed and transferred to a $35 \mathrm{~mm}$ petri dish with fresh mapping solution. The dish was placed on a stage heated to $37^{\circ} \mathrm{C}$ for the remainder of the experiment. hiPSC-CM monolayers were stimulated from one edge with a palladium line electrode delivering $10 \mathrm{~V}$, $10 \mathrm{~ms}$ monophasic rectangular pulses at predetermined cycle lengths: $1000 \mathrm{~ms}, 700 \mathrm{~ms}$, and $500 \mathrm{~ms}$. Optical mapping recordings were taken using a $100 \times 100$ pixel CMOS camera (MiCAM Ultima-L; SciMedia, Costa Mesa, CA, USA) and analyzed using custom MATLAB scripts (Version R2019b, MathWorks, Natick, MA, USA; details in Supplementary Methods).

\section{6. siRNA Transfection}

Pre-designed siRNA oligos targeted against DSG2 were purchased (MilliporeSigma), and a cocktail of three distinct oligos (see Table S2 for oligo sequences) was complexed for transfection using TransIT-siQUEST transfection reagent (Mirus Bio, Madison, WI, USA). On differentiation day 35, hiPSC-CM media was replaced with B27+ media supplemented with siRNA complexes for a final concentration of $50 \mathrm{nM}$. $48 \mathrm{~h}$ later, the media was replaced with fresh B27+ media, and the samples were analyzed the following day (72 $\mathrm{h}$ total treatment time).

\subsection{Statistics}

Unless otherwise indicated, all data are presented as mean $\pm 95 \%$ confidence interval, calculated in R. Continuous variables were compared using unpaired, unequal variance Student's $t$-test with a Holm-Bonferroni family-wise error correction. $p<0.05$ in two-tailed analysis was considered significant. For RT-qPCR data, normalized relative quantities and their standard errors were calculated using the EasyqpcR software package (Version 1.34) [37].

\section{Results}

\subsection{Family History and Clinical Phenotypes of ARVC Patient Donor}

The patient (identified as JHU013) is a 48-year-old woman of South Asian descent who harbors a familial genetic variant in DSG2 (Figure 1A). Her 12-lead electrocardiogram 
(ECG) was positive for T wave inversions (initially observed at age 22; Figure 1B) and consistently negative for epsilon waves. She began experiencing paroxysmal dizziness and palpitations at age 32, but she was not diagnosed with ARVC until age 45 when a diagnosis in her sister led to a thorough cardiac evaluation. Specifically, her ECG showed T wave inversions in anterior precordial leads V1-V4 with inferior repolarization abnormalities in leads II, III, and aVF (Figure 1B, Table 1). Cardiac magnetic resonance (CMR) imaging demonstrated mild dilation of the right ventricle and dyskinesia of the right ventricular free wall $\left(108 \mathrm{~mL} / \mathrm{m}^{2} \mathrm{RV}\right.$ end-diastolic volume to body surface area; Table 1, Figure S1) with microaneurysm formation along the right ventricular outflow tract. CMR was also notable for extensive delayed gadolinium enhancement involving the right ventricular free and inferior walls, suggesting significant fibrosis in those regions. However, no ventricular wall fat infiltration was observed. As of last follow-up, the hiPSC donor had no documented history of sustained ventricular arrhythmia. Further familial screening revealed several carriers of the DSG2 c.2358delA variant, including the donor, her sister (proband), nephew, niece, her mother, and both of her children (Figure 1A). At last follow-up, none of the children of the donor or her sister who carry the variant (ages 13-20) had been diagnosed with ARVC, though they are on the low end of the age range at which symptoms typically present. However, the younger family members have experienced some notable cardiac abnormalities. A Holter monitor revealed 420 premature ventricular complexes (PVCs) in the proband's son (age 20) over a 24-hour period, which was high but does not reach the 2010 Task Force criteria of $\geq 500$ PVCs over $24 \mathrm{~h}$. The proband's daughter (age 17) has a history of palpitations, and one of the donor's daughters (age 16) has a history of palpitations as well as $\mathrm{T}$ wave inversions in V1 and V3 on ECG.

A

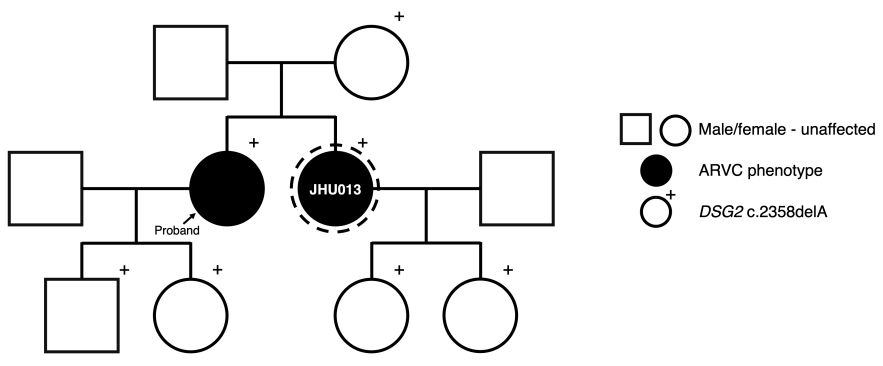

B

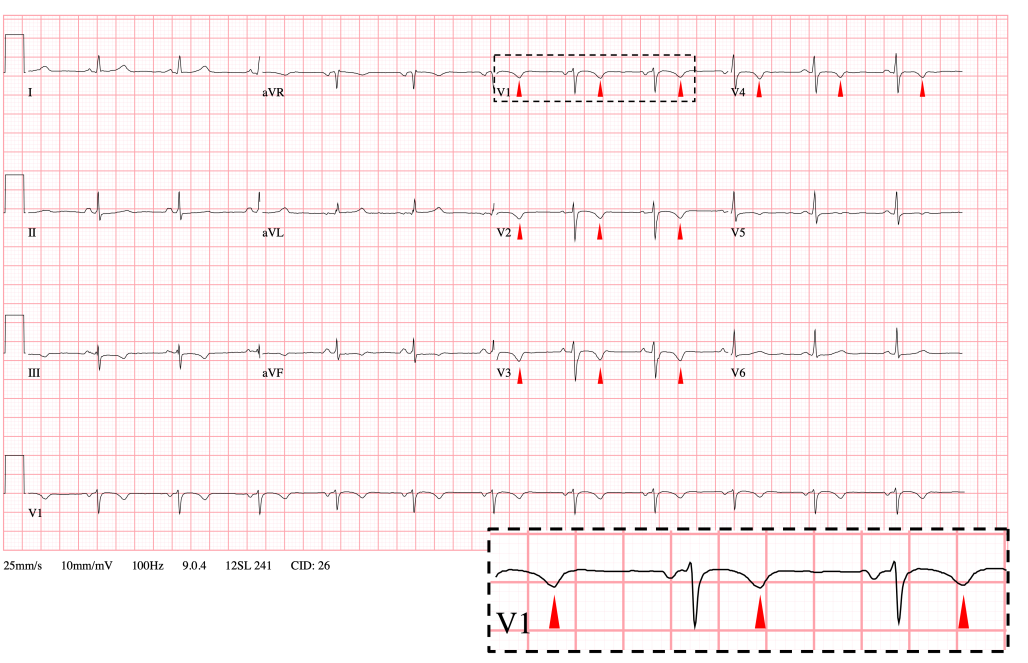

Figure 1. Family pedigree and electrocardiogram from hiPSC donor. (A) Family pedigree of patient JHU013 (dashed circle) harboring a pathogenic variant in desmoglein-2 (DSG2 c.2358delA). Arrow signifies proband. (B) 12-lead ECG of patient JHU013 showing T wave inversion in leads V1-V4 (red markers), a classic feature in ARVC patients. Dashed box inset, highlighting T wave inversion in lead V1. 
Table 1. Demographic data and clinical history of patient JHU013. 2010 Task Force Criteria were used.

\begin{tabular}{|c|c|}
\hline Attribute & Finding \\
\hline \multicolumn{2}{|l|}{ Demographic } \\
\hline Gender & Female \\
\hline Race/Ethnicity & South Asian \\
\hline Presentation (age [yrs]: symptoms) & $\begin{array}{l}\text { 22: anterior precordial T wave inversions noted on ECG. } \\
\text { 32: dizziness, palpations }\end{array}$ \\
\hline Age at diagnosis (yrs) & 45 \\
\hline Age at last follow-up (yrs) & 51 \\
\hline \multicolumn{2}{|l|}{2010 Task Force Criteria Fulfillment [26] } \\
\hline ECG Repolarization Abnm. & $\begin{array}{l}\text { Major: Inverted } T \text { waves in right precordial leads }\left(V_{1}, V_{2} \text {, }\right. \\
\left.\text { and } V_{3}\right)\end{array}$ \\
\hline ECG Depolarization Abnm. & None \\
\hline Arrhythmia & Minor: $>500$ PVCs per $24 \mathrm{~h}$ on Holter monitor \\
\hline Structural & Major: Regional RV dyskinesia + RV EDVi > 100 mL/m² \\
\hline Family History & $\begin{array}{c}\text { Major: ARVC confirmed in a first-degree relative who meets } \\
\text { current Task Force criteria }\end{array}$ \\
\hline \multicolumn{2}{|l|}{ Cardiac MRI (at last follow-up) } \\
\hline $\mathrm{RV} \operatorname{EDVi}\left(\mathrm{mL} / \mathrm{m}^{2}\right)$ & 118 \\
\hline RV EDVi/LV EDVi & 2.62 \\
\hline RV wall-motion Abnm. & Regional dyskinesia, aneurysms \\
\hline LV wall-motion Abnm. & None \\
\hline RVEF (\%) & 44 \\
\hline $\operatorname{LVEF}(\%)$ & 57 \\
\hline Delayed enhancement & $\begin{array}{l}\text { Extensive delayed enhancement in RV free wall and inferior } \\
\text { wall }\end{array}$ \\
\hline \multicolumn{2}{|l|}{ Events } \\
\hline Sustained ventricular arrythmia & No \\
\hline Cardiac transplant & No \\
\hline Death & No \\
\hline
\end{tabular}

Abnm.: abnormalities, RV: right ventricle, LV: left ventricle, RVEF: right ventricular ejection fraction, LVEF: left ventricular ejection fraction, EDVi: end diastolic volume indexed to body surface area.

\subsection{Establishment of Patient-Specific Human Induced Pluripotent Stem Cell Line with Novel Dsg2 Pathogenic Variant}

Sequence analysis shows that the DSG2 variant harbored by patient JHU013 - listed in ClinVar as NM_001943.5(DSG2):c.2358del (p.Asp787fs) - causes a frameshift mutation, resulting in the formation of a premature stop codon, 21 codons downstream. This frameshift produces a truncation in the intracellular cadherin-like domain (ICD) of the DSG2 protein (Figure 2A). In line with standards of the American College of Medical Genetics and Genomics [38,39], this variant has been classified as likely pathogenic. Cascade screening and clinical criteria are consistent with the DSG2 loss of function contributing to the pathogenesis of both the proband and JHU013. 
A

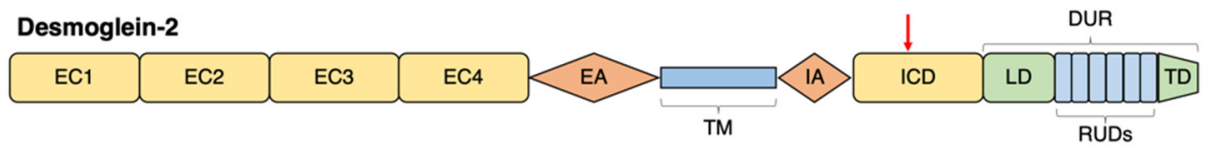

B

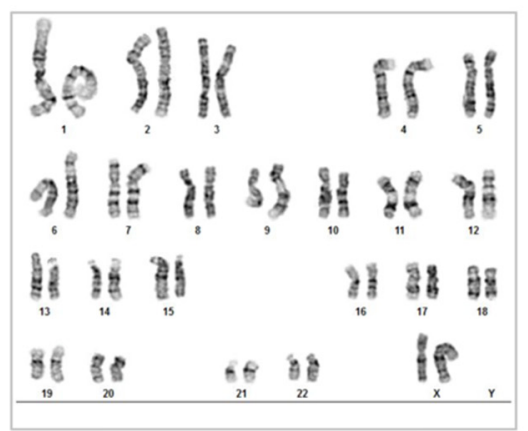

D

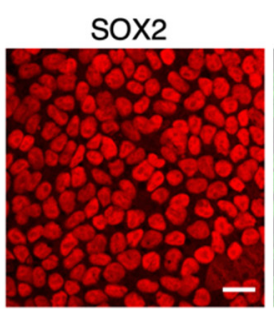

C
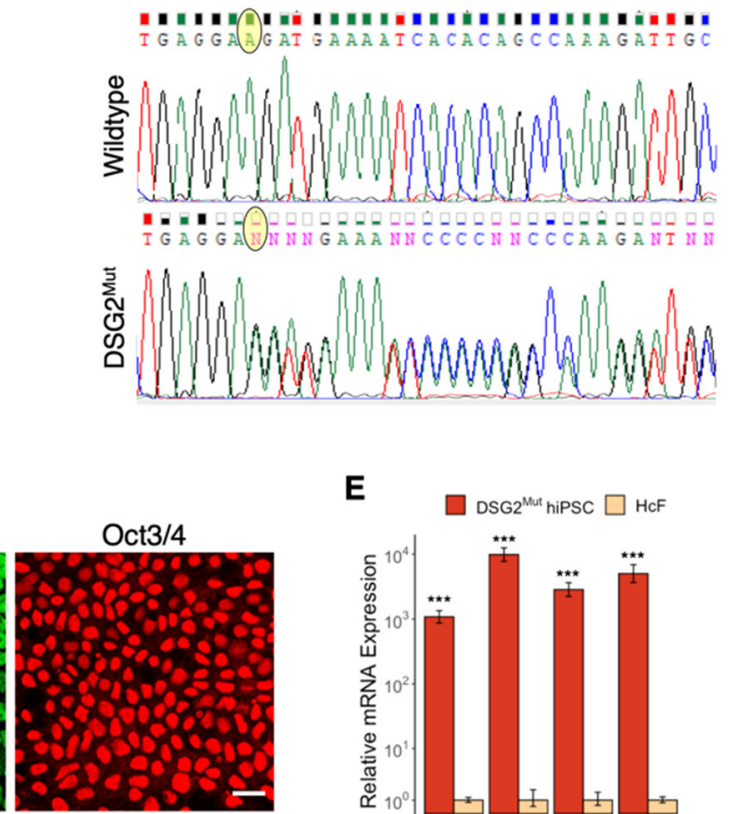

E

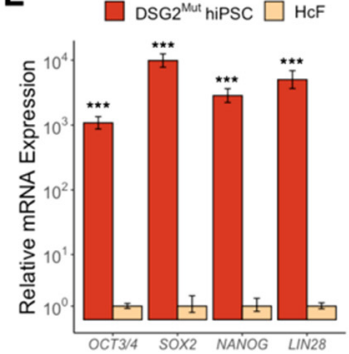

Figure 2. Donor-derived hiPSC line harbors heterozygous DSG2 c.2358delA variant and expresses markers of pluripotency. (A) Schematic protein structure of desmoglein-2. EC1-4, extracellular cadherin domains; EA, extracellular anchor domain; TM, transmembrane domain; IA, intracellular anchor domain; ICD, intracellular cadherin-like domain; LD, linker domain; RUD, repeat unit domain with 6 repeats; TD, terminal domain; DUR, DSG unique region; red arrow indicates location of truncation caused by the DSG2 c.2358delA variant. (B) Chromosomal analysis of JHU013 (DSG2 ${ }^{\text {Mut }}$ ) hiPSCs. (C) Sanger sequencing of DSG2 in control and DSG2 ${ }^{\text {Mut }}$ hiPSCs showing the c.2358delA mutation. Yellow oval highlights c.2358 nucleotide under investigation. ' $\mathrm{N}$ ' indicates likely heterozygosity at that location. (D) Confocal images of DSG2 ${ }^{\mathrm{Mut}}$ hiPSCs depicting immunopositivity for canonical markers of pluripotency: Sex determining region Y-box 2 (SOX2), homeobox NANOG, and Octamer binding transcription factor-3/4 (OCT3/4). (E) RT-qPCR analysis showed abundant expression of pluripotency-associated transcripts in $D S G 2^{\mathrm{Mut}}$ hiPSCs relative to terminally differentiated control cells (primary neonatal human cardiac fibroblasts, $\mathrm{HcF}) . n=3$ for each group. ${ }^{* * *} p<0.001$.

In order to determine the functional and pathological consequences of the DSG2 c.2358delA variant, we generated an hiPSC line from the affected patient. Briefly, peripheral blood mononuclear cells were isolated from the donor's blood, induced to form blast cells and reprogrammed into pluripotency via transfection with Sendai virus vectors containing the Yamanaka factors. These cells were expanded, and clonal colonies were selected and characterized. Chromosomal analysis of this new DSG2-mutant (DSG2 ${ }^{\text {Mut }}$ ) hiPSC line demonstrated a normal karyotype, and Sanger sequencing showed a heterozygous DSG2 c.2358delA variant present in the reprogrammed cells (Figure 2B,C). Canonical markers of pluripotency were verified via immunofluorescent signal and RT-qPCR in DSG2 ${ }^{\text {Mut }}$ hiPSCs (Figure 2D,E).

\subsection{Biomolecular Characterization of DSG2 $2^{\text {Mut }}$ hiPSC-CM Model}

For controls, we selected an hiPSC line derived from a healthy volunteer of the same gender and similar age following an identical protocol (JHU001, "Ctrl1"; female), as well as a widely available wildtype control line (WTC11, "Ctrl2"; male). hiPSCs from all three lines were subjected to the same Wnt-mediated cardiogenic differentiation protocol and 
began to beat on approximately day 7 . On days 10-14 the CM population was enriched metabolically via lactate purification as previously described. Cardiomyocytes formed a syncytial monolayer after being plated onto coverslips on day 28 and were cultured until day 35-39, when they were analyzed. Differentiated hiPSC-CMs from all three lines were measured by flow cytometry to be $>90 \%$ positive for cardiac troponin T on day 35 , indicating high purity of cardiomyocytes, with no differences in differentiation efficiency noted between control and DSG2 ${ }^{\text {Mut }}$ hiPSC lines (Figure S2). The mean spontaneous beating rates for the hiPSC-CM monolayers measured in culture was similar among all lines on differentiation day $38\left(67.6 \pm 5.7\right.$ beats per minute in $D S G 2^{\text {Mut }}$ vs. $68.4 \pm 12.1 \mathrm{BPM}$ in Ctrl1 and $64.8 \pm 5.8$ BPM in Ctrl2; $n=14,12$, and 12, respectively).

We first characterized the expression and localization of key desmosomal proteins and cardiac ion channels in the DSG2-mutant hiPSC-CM model. Specifically, RT-qPCR analysis showed that mRNA expression of DSG2 is significantly reduced in the mutant hiPSC-CMs as compared to both Ctrl1 and Ctrl2 (35.2\% and $49.6 \%$ reduction, respectively) (Figure $3 \mathrm{~A})$, using a primer targeted to a site upstream of the DSG2 c.2358delA location. Sodium and potassium channel genes $S C N 5 A$ and $K C N Q 1$, respectively, were more highly expressed in $D S G 2^{\text {Mut }} \mathrm{CMs}$ compared to $\mathrm{Ctrl1}$, but not $\mathrm{Ctrl2}$, as was $\mathrm{Ca}^{2+}$-handling gene CAMK2A. In other $\mathrm{Ca}^{2+}$-handling genes, we observed increased $R Y R 2$ expression and reduced CASQ2 and SLN expression in DSG2 ${ }^{\text {Mut }}$ CMs compared to controls.

A
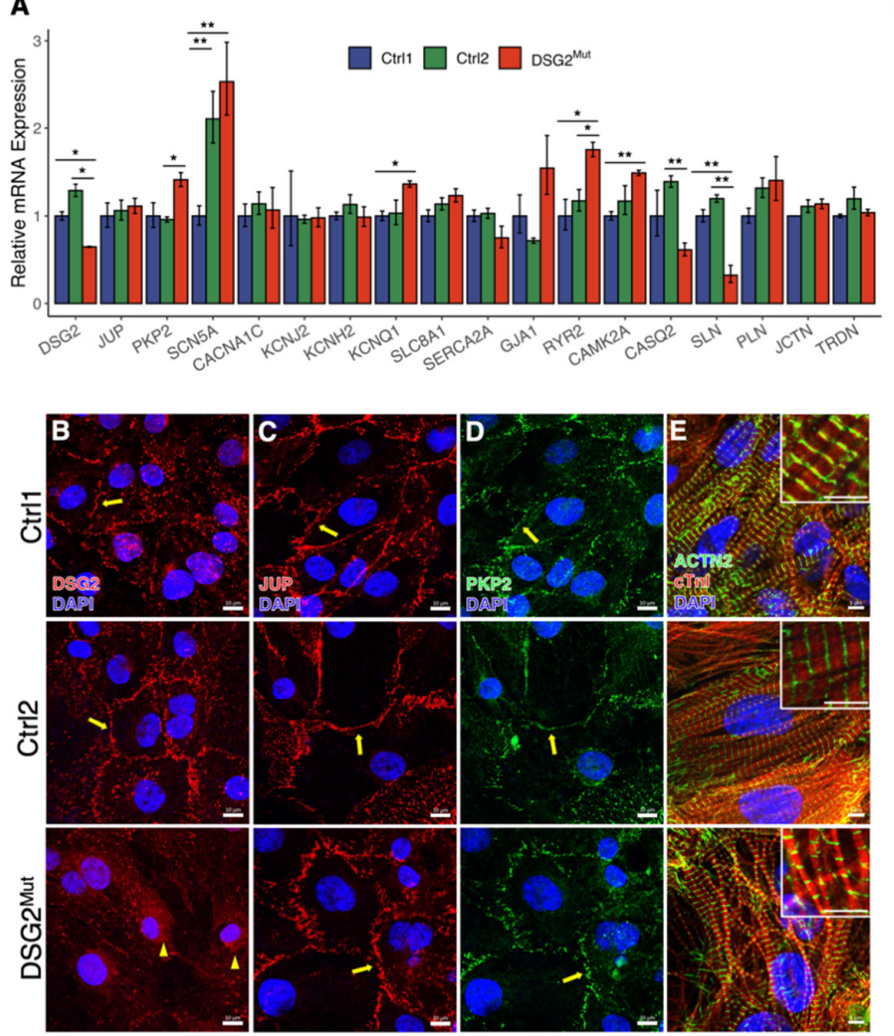

$\mathbf{F}$

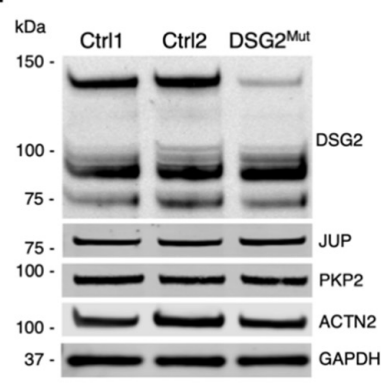

G

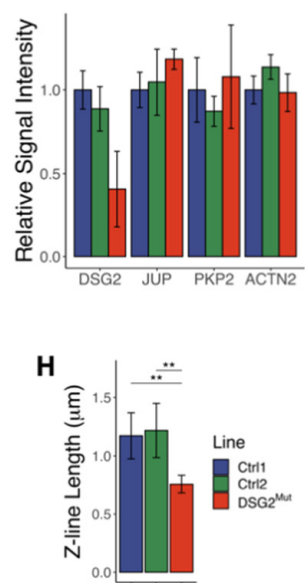

Figure 3. RT-qPCR, immunostaining and Western blot analyses of DSG2 ${ }^{\text {Mut }}$ hiPSC-CMs. (A) RT-qPCR analysis of normal and DSG2 ${ }^{\text {Mut }}$ hiPSC-CMs, normalized to Ctrl1. DSG2 primer targets a region upstream of the c. 2358 deletion. $n=3$ for all groups. (B-E) Representative control and DSG2 ${ }^{\text {Mut }}$ hiPSC-CMs immunostained for desmosomal and structural proteins. Yellow arrows highlight presence of desmosomal proteins at cell-cell boundaries; yellow arrowheads denote perinuclear localization of DSG2. White scale bars: $10 \mu \mathrm{m}$ (B-D), $5 \mu \mathrm{m}(\mathbf{E})$. (F) Whole lysate western immunoblots of DSG2 ${ }^{\text {Mut }}$ and control hiPSC-CMs, probed for desmosomal proteins and $\alpha$-actinin (ACTN2). (G) Quantification of western blot signal intensity relative to GAPDH and normalized to Ctrl1. DSG2 was quantified using the full-length $130 \mathrm{kDa}$ band. $n=3$ for each line. (H) Z-line lengths quantified from confocal images of $\alpha$-actinin immunostaining. Shorter Z-lines in DSG2 ${ }^{\mathrm{Mut}}$ cells correspond to narrower myofibrils. Two regions were randomly selected for quantification from three unique images of each line. ${ }^{*} p<0.05 ;{ }^{* *} p<0.01$. 
Immunofluorescent imaging illustrated reduced overall signal intensity of desmoglein2 , but also revealed reduced localization at the cell membrane in conjunction with perinuclear accumulation in DSG2 ${ }^{\text {Mut }}$ hiPSC-CMs (Figure 3B). The latter finding suggests defects in trafficking of desmoglein-2 in these cells, as previously reported in human and animal models of ARVC $[40,41]$. Western immunoblot analysis demonstrated much lower expression of full-length $(\sim 130 \mathrm{kDa})$ desmoglein-2 in $D S G 2^{\text {Mut }} \mathrm{CMs}$, but retained lower molecular weight bands (Figure 3F,G).

We found mRNA abundance of desmosomal components PKP2 and JUP to be slightly increased and unaffected, respectively, in DSG2 ${ }^{\text {Mut }}$ cells, but observed no difference in protein localization of either marker by immunostaining (Figure 3C,D) or expression by Western blot studies (Figure 3F,G). We also measured the abundance of two key calcium handling proteins, CaM Kinase II and PKA-C in their active (i.e. phosphorylated) forms (pCaMKII and pPKA-C) and found pCaMKII abundance was not significantly different among the three lines. DSG2 ${ }^{\text {Mut }}$ hiPSC-CMs had levels of pPKA-C similar to those in Ctrl1, but greater than those in Ctrl2 (Figure S3). Immunostaining for $\alpha$-actinin and cardiac troponin I revealed that DSG2 ${ }^{\text {Mut }}$ hiPSC-CMs displayed narrower myofibrils compared to control hiPSC-CMs (Figure 3E), although Western blots demonstrated that $\alpha$-actinin protein abundance was not altered in $D S G 2^{\text {Mut }}$ CMs. To assess myofibril diameter, we measured the length of Z-lines, denoted by $\alpha$-actinin immunopositivity, in randomly selected regions across several 100X confocal images. We found myofibril diameter in both control lines to be in agreement with measurements reported in the literature [42] but confirmed that Z-line length, i.e. myofibril width, was significantly shorter in DSG2 ${ }^{\text {Mut }}$ CMs compared to controls $\left(0.76 \pm 0.08 \mu \mathrm{m}\right.$ in $D S G 2^{\text {Mut }}$ vs. $1.17 \pm 0.2 \mu \mathrm{m}$ in Ctrl1 and $1.22 \pm 0.23 \mu \mathrm{m}$ in Ctrl2; Figure $3 \mathrm{H})$.

\subsection{Cytokine and Chemokine Expression in DSG2 ${ }^{\text {Mut }}$ hiPSC-CMs}

Myocardial inflammation is a recurrent pathological hallmark of ARVC, even presenting as myocarditis before subsequent genetic screening confirms a diagnosis of ARVC [43]. Considering we previously showed that PKP2-mutant hiPSC-CMs produce and secrete potent inflammatory cytokines via nuclear factor kappa-B (NFkB)-mediated transcription [15], we assessed both hiPSC-CM lysates and cell culture supernatants for the production and secretion, respectively, of more than 100 cytokines in DSG2 ${ }^{\text {Mut }}$ and control cell lines. We identified eight cytokines in DSG2 ${ }^{\text {Mut }}$ hiPSC-CMs that are involved in the innate immune response and complement component system that were upregulated in DSG2 ${ }^{\text {Mut }}$ hiPSC-CM lysates (Figure $4 \mathrm{~A}-\mathrm{C}, \mathrm{H}$ ). Included among these was the receptor for advanced glycation end products (RAGE), which binds to nuclear high-mobility group box-1 (HMGB1) to promote cell death [44]; this is consistent with our recent work demonstrating that HMGB1nuclear loss leads to myocyte cell necroptosis in Dsg2-mutant mice [14]. 

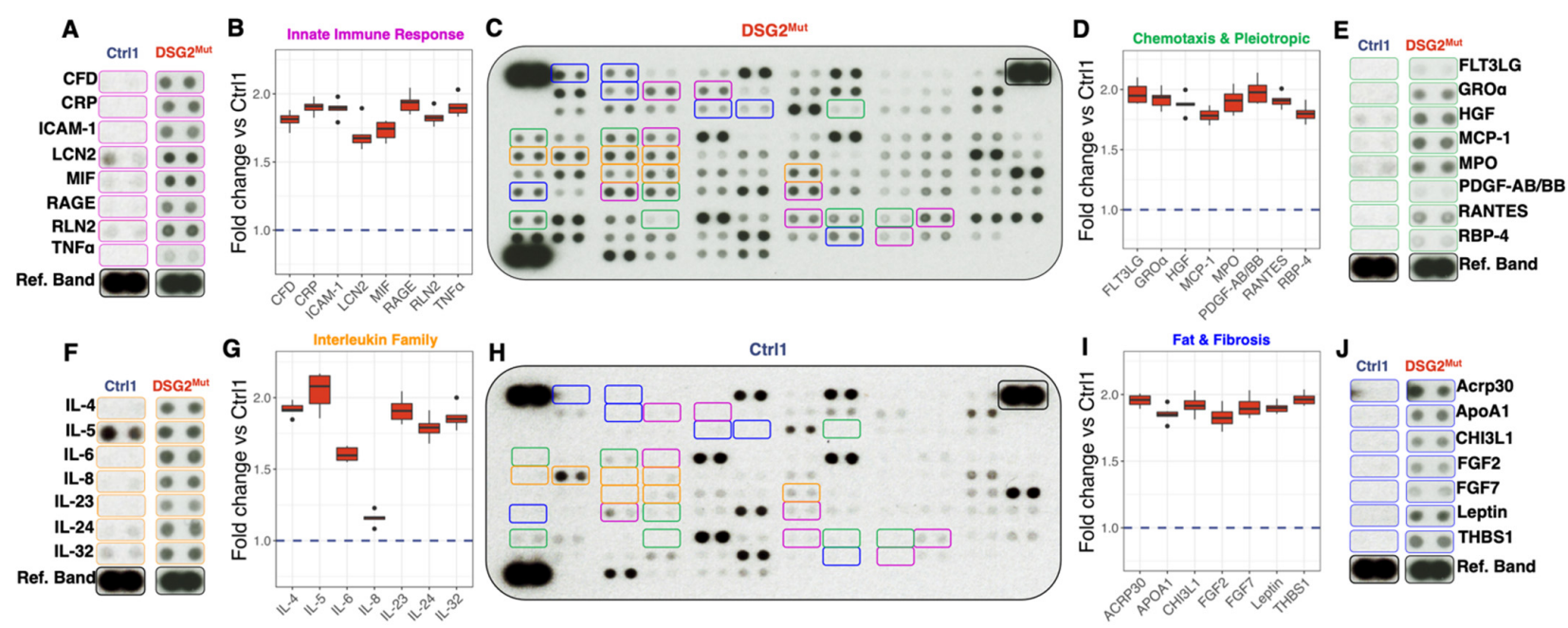

Figure 4. Cytokine expression in DSG2 ${ }^{\text {Mut }}$ hiPSC-CMs. (A,C,E,F,H,J) Representative cytokine immunoblots from wildtype control (Ctrl1) and DSG2 ${ }^{\text {Mut }}$ hiPSC-CMs, grouped based on their role in inflammation: (A) the innate immune response (purple); (E) chemoattractant and pleiotropic regulation (green); (F) interleukins that regulate immune cell differentiation and proliferation (orange); and (J) adipo-fibrokines involved in lipid accumulation and metabolism and fibrotic remodeling (blue). (B,D,G,I) Box and whisker plots of selected cytokines from Figure 4A,E,F,J. Data presented as mean \pm standard deviation.; Blue dashed line represents normalized values for Ctrl1; $C \operatorname{trl1}(n=4)$; DSG2 ${ }^{\text {Mut }}(n=6)$; For all values shown in $(\mathbf{B}, \mathbf{D}, \mathbf{G}, \mathbf{I}) p<0.05$ comparing $D S G 2^{\text {Mut }}$ vs. Ctrl1.

We identified numerous upregulated pleiotropic cytokines and chemokines involved in mobilizing and recruiting immune cells (Figure $4 \mathrm{D}, \mathrm{E}$ ), and members of the potent, proinflammatory interleukin (IL)-family (Figure 4F,G). Of these, monocyte chemoattract protein-1 (MCP-1) was upregulated in DSG2 ${ }^{\text {Mut }}$ hiPSC-CMs, a finding previously reported in Dsg2-mutant mouse myocardium and in hiPSC-CMs and cell culture supernatant from PKP2-mutant hiPSC-CMs [15]. Of the members of the IL-family, cytokines that regulate immune cell differentiation (e.g., IL-5, IL-6, and IL-32) and proliferation (e.g., IL-4, IL-23, IL24) were elevated in $D S G 2^{\text {Mut }}$ CMs (Figure $4 F, G$ ). Lastly, several adipo-fibrokines that drive lipid accumulation, regulate adipocyte survival and proliferation, and promote fibrotic remodeling were significantly elevated in $D S G 2^{\text {Mut }}$ hiPSC-CMs compared to controls (Figure 4I,J); however, Oil Red O staining did not reveal lipid deposition in mutant or control hiPSC-CMs (Figure S4).

\subsection{Immune Signaling in DSG2 $2^{\text {Mut }}$ hiPSC-CMs Is Regulated by Canonical and Non-Canonical NFkB Pathways}

Our prior studies demonstrated that both myocardial inflammation in Dsg2-mutant mice and cytokine production in PKP2-mutant hiPSC-CMs are regulated via the canonical NFkB pathway [15]. We performed bulk RNA-Seq (Figure 5A) from normal and DSG2 ${ }^{\text {Mut }}$ hiPSC-CMs and applied KEGG PATHWAY analysis to map out the components of the NFkB pathway (Figure 5B). Our results implicate four cell surface receptors involved in canonical and non-canonical NFkB signaling that were upregulated in DSG2 ${ }^{\text {Mut }}$ CMs: IL1R, XEDAR, Toll-like receptor-4 (TLR4), and CD40 (Figure 5A,B). TLR4 is a pleiotropic NFkB transcription factor regulating IL-23-induced T-cell infiltration [45], a cytokine we also found upregulated in DSG2 ${ }^{\text {Mut }}$ hiPSC-CMs (Figure 4F,G). Downstream of TLR4- and IL1R-induced NFkB activation, we found IL1R kinase-1 (IRAK1) and TRIF to also be upregulated in DSG2 ${ }^{\text {Mut }}$ CMs (Figure 5A,B). These two pathways merge to signal through TGF $\beta$ activating kinase (TAK1/MAP3K7) and TAK binding protein (TAB) to activate p50/p65 (RELA) canonical NFkB (Figure 5A-C). While RELA itself was not differentially expressed via RNA-Seq analysis, RELA-mediated transcripts (e.g., IL8, COX4, Figure 5A,B) were upregulated in DSG2 ${ }^{\text {Mut }}$ hiPSC-CMs. This discrepancy is potentially explained by regulatory differences imposed by post-transcriptional mechanisms. 

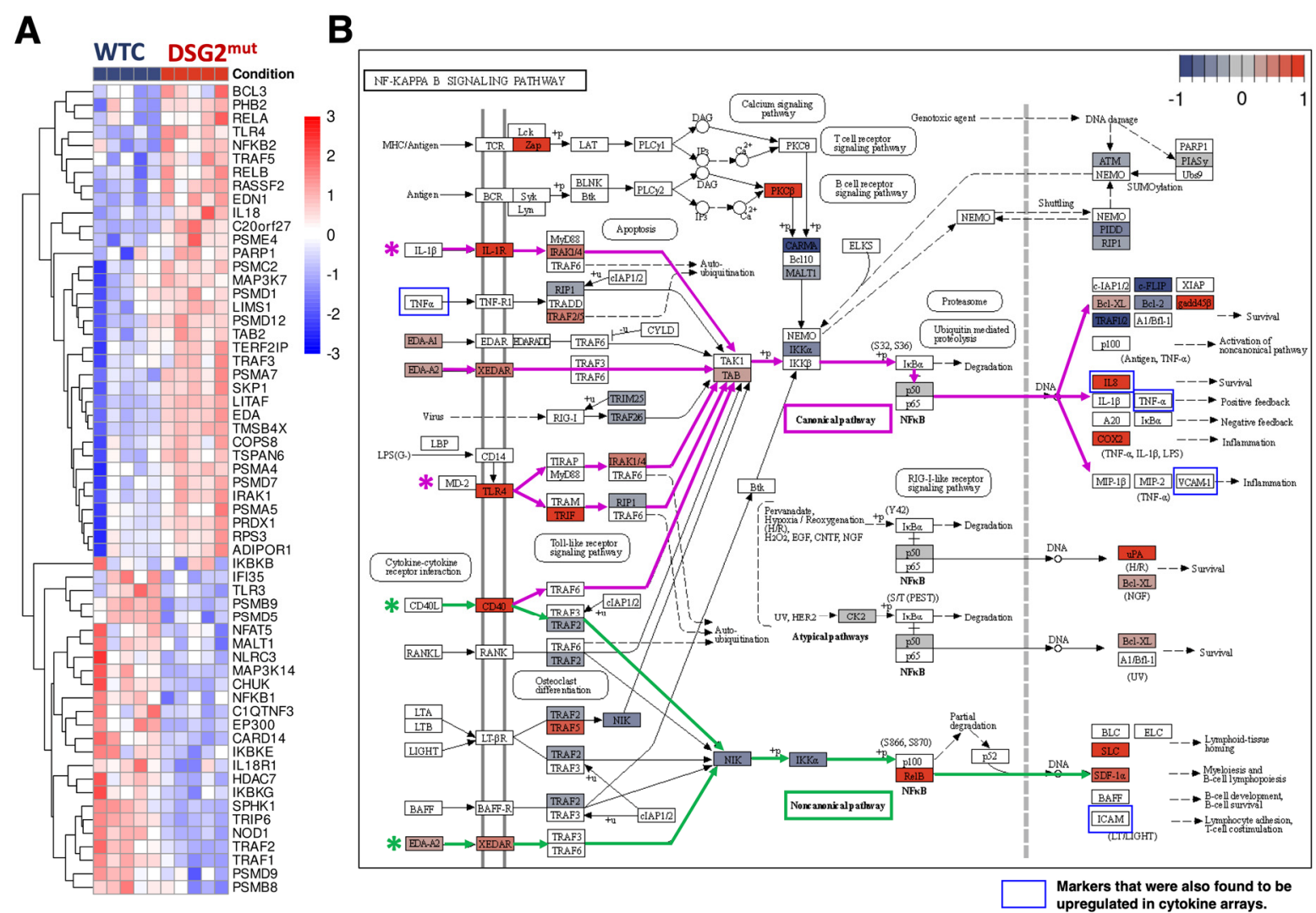

C Canonical NFKB

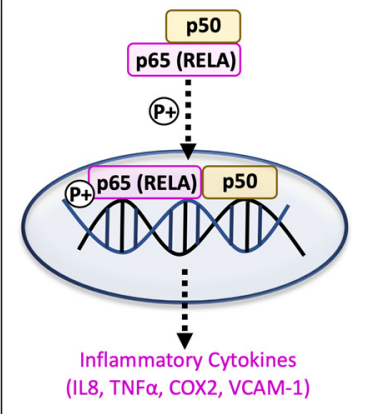

Non-canonical NFKB

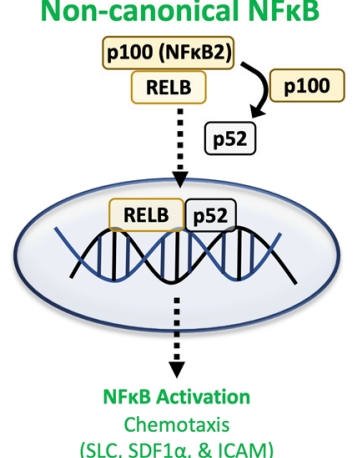

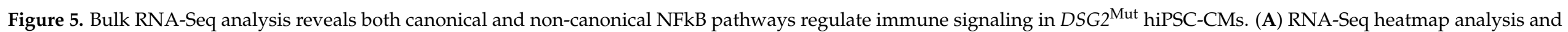

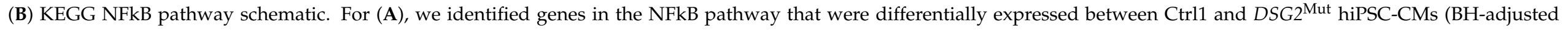

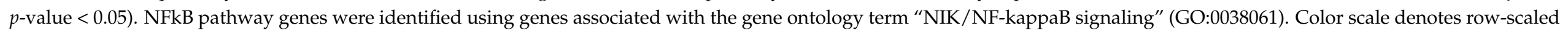

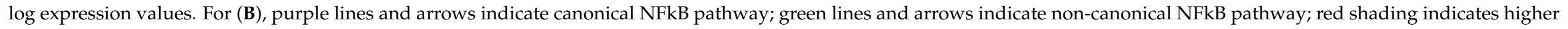

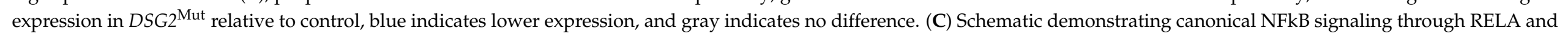
non-canonical NFkB signaling through RELB. 
We next focused our attention to the non-canonical cell surface receptors, CD40 and XEDAR. Non-canonical NFkB signaling is mediated through tumor necrosis factor receptorassociated factors (TRAF1-6), where prior work demonstrated TRAF2 acts as a negative regulator of NFKB [46-48]. Our data showing reduced levels of TRAF2 (Figure 5A,B), and upregulation of RELB, a positive regulator of non-canonical NFkB signaling (Figure 5A,B), is consistent with the role of non-canonical NFkB signaling in this model of ARVC. Furthermore, while not significantly differentially expressed, NFkB1 (p105) and TRIP6 (NFkB suppressors) were both downregulated in DSG2 ${ }^{\mathrm{Mut}}$ hiPSC-CMs (Figure 5A,B).

\subsection{Shortened Action Potential Durations, Heterogeneous Excitation, and Altered Calcium Handling in DSG2 ${ }^{\mathrm{Mut}} \mathrm{CMs}$}

On day 28 of differentiation, hiPSC-CMs from each line were plated onto coverslips and incubated for 9-11 days to allow a confluent monolayer to develop. At day 37-39, optical mapping recordings were taken under electrical pacing to measure the action potential and calcium transient characteristics of the monolayers. Action potential duration at $80 \%$ repolarization $\left(\mathrm{APD}_{80}\right)$ was significantly reduced in $D S G 2^{\mathrm{Mut}} \mathrm{CMs}$ compared with both control lines $\left(220 \pm 24 \mathrm{~ms}\right.$ in DSG2 ${ }^{\text {Mut }}$ vs. $311 \pm 27 \mathrm{~ms}$ in Ctrl1 and $365 \pm 26 \mathrm{~ms}$ in Ctrl2 at $1000 \mathrm{~ms}$ pacing $)$ as was $\mathrm{APD}_{30}\left(134 \pm 15 \mathrm{~ms}\right.$ in $D S G 2^{\text {Mut }}$ vs. $213 \pm 21 \mathrm{~ms}$ in Ctrl1 and $227 \pm 25 \mathrm{~ms}$ in Ctrl2 at $1000 \mathrm{~ms}$ pacing; Figure 6A,B), revealing important differences in repolarization. A possible basis for the APD differences is suggested by RT-qPCR analysis, which showed that mRNA expression of KCNQ1 was higher in the DSG2 ${ }^{\mathrm{Mut}} \mathrm{CMs}$ compared to Ctrl1 (Figure 3A). KCNQ1 encodes the ion channel that mediates $I_{K s}$, a key repolarizing current in cardiomyocytes.

Optical mapping experiments showed similar conduction velocities and conduction heterogeneity in all lines (Figure 6D,E) with no significant differences in relative upstroke velocity (Figure 6C). However, we did find that the upstroke velocity in the DSG2 ${ }^{\text {Mut }}$ hiPSC-CMs was more variable across the monolayer within each sample $(0.27 \pm 0.1$ relative standard deviation (RSD) in DSG2 ${ }^{\text {Mut }}$ vs. $0.15 \pm 0.01$ in Ctrl1 and $0.13 \pm 0.06$ in Ctrl2 at $1000 \mathrm{~ms}$ pacing; Figure 6C). This suggests substantial spatial heterogeneity of excitability or electrical coupling in these cells, though the latter seems less likely because of the lack of measurable differences in conduction heterogeneity (Figure 6E).

In addition, mapping the relative intracellular calcium concentration in hiPSC-CM monolayers during excitation revealed significant differences in calcium dynamics. Time to peak $\mathrm{Ca}^{2+}$ was considerably shorter in $D S G 2^{\text {Mut }} \mathrm{CMs}$ than in controls $(98 \pm 18 \mathrm{~ms}$ in $D S G 2^{\text {Mut }}$ vs. $170 \pm 40 \mathrm{~ms}$ in Ctrl1 and $183 \pm 32 \mathrm{~ms}$ in Ctrl2; Figure 6G). At faster pacing rates $D S G 2^{\text {Mut }} \mathrm{CMs}$ also displayed slowed $\mathrm{Ca}^{2+}$ transient decay compared to controls (Figure 6H), though this effect was not observed at 1000 ms pacing. Notably, RT-qPCR analysis demonstrated significant differences in expression of several calcium handling genes in $D S G 2^{\text {Mut }}$ hiPSC-CMs - namely, increased mRNA expression of ryanodine receptor $2(R Y R 2)$ along with lower expression of sarcolipin $(S L N)$ and calsequestrin (CASQ2) (Figure 3A). 
A

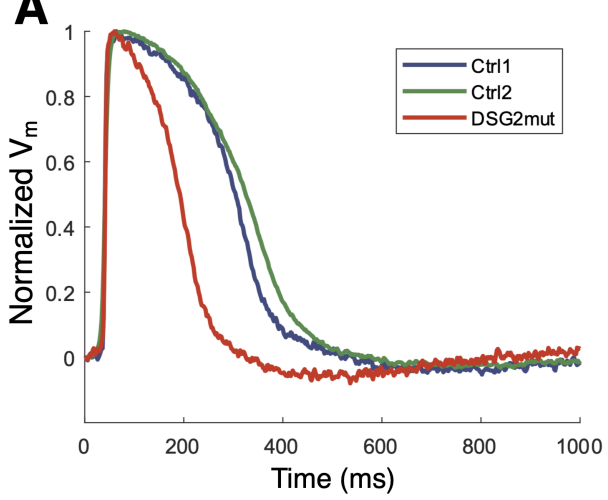

B

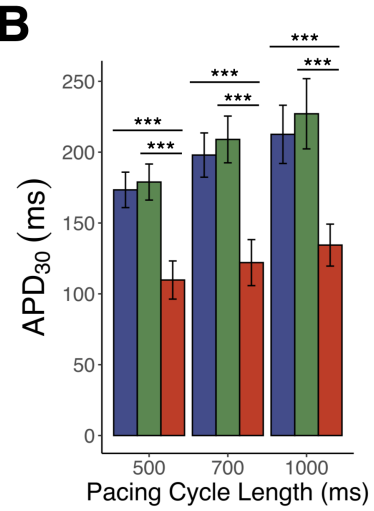

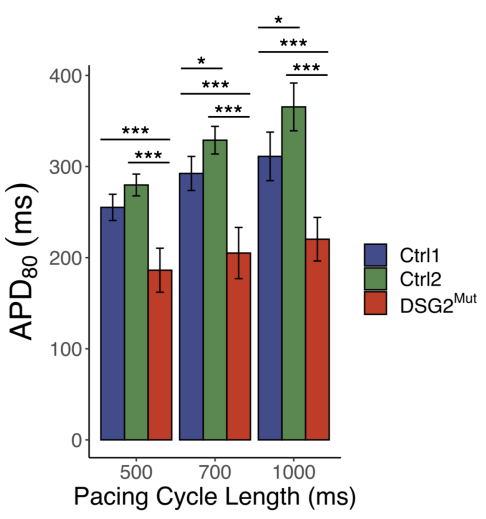

$\mathbf{E}$
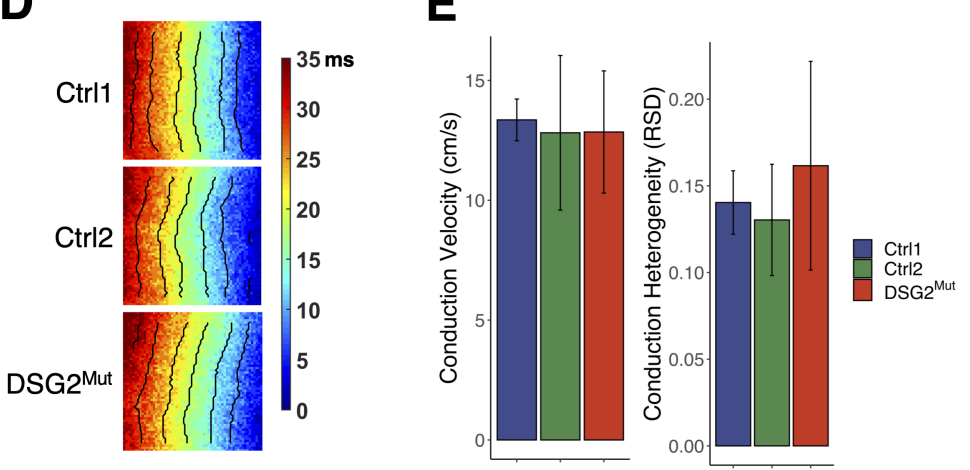
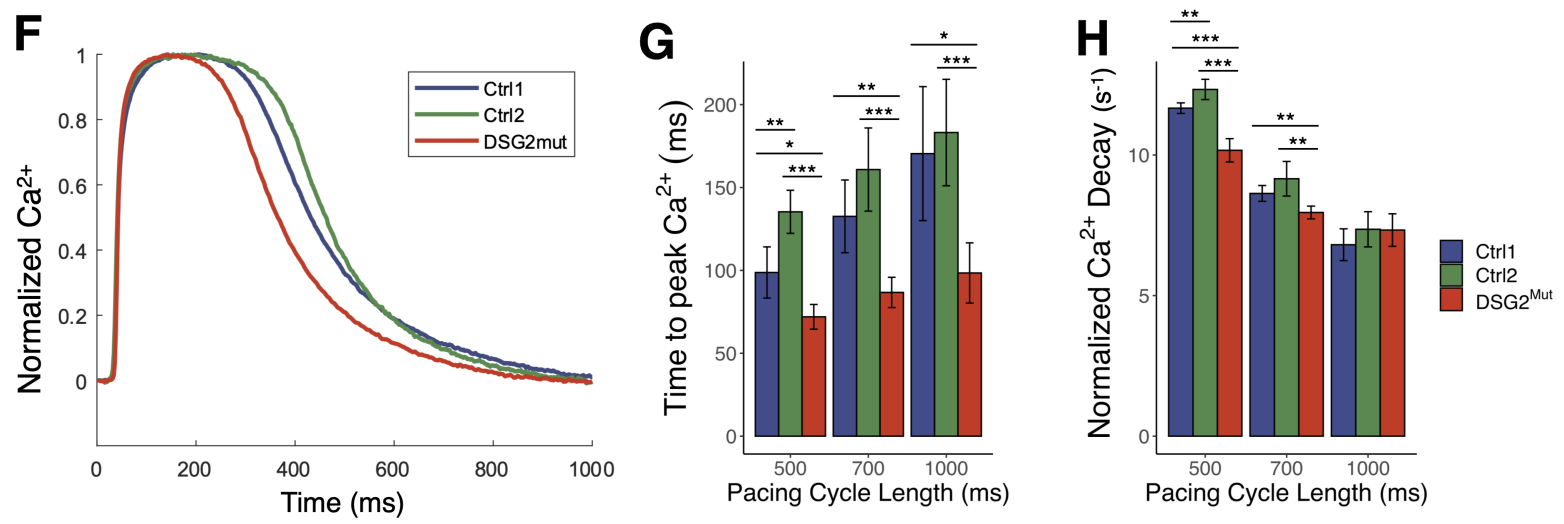

Figure 6. Action potential characteristics of hiPSC-CM monolayers measured via optical mapping. (A) Representative traces of normalized transmembrane potential $\left(\mathrm{V}_{\mathrm{m}}\right)$. (B) Action Potential Duration (APD) measured at $30 \%$ and $80 \%$ repolarization. (C) Relative upstroke velocity measured at $1000 \mathrm{~ms}$ pacing and spatial heterogeneity of upstroke velocity, calculated as the relative standard deviation (RSD) of all measurements within each sample. (D) Activation maps with isochrone lines spaced $5 \mathrm{~ms}$ apart. (E) Mean conduction velocity and conduction heterogeneity measurements at $1000 \mathrm{~ms}$ pacing. (F) Representative normalized calcium traces. (G) Time-to-peak $\mathrm{Ca}^{2+}$. (H) $\mathrm{Ca}^{2+}$ decay rate, measured using normalized data. $\mathrm{V}_{\mathrm{m}}$ mapping (B-E): Ctrl1 $(n=8), \operatorname{Ctrl} 2(n=13) D S G 2^{\mathrm{Mut}}(n=9) . \mathrm{Ca}^{2+}$ mapping $(\mathbf{G}, \mathbf{H}): \mathbf{C} \operatorname{trl} 1(n=10), \mathrm{Ctrl} 2(n=7), D S G 2^{\mathrm{Mut}}(n=9)$. ${ }^{*} p<0.05 ;{ }^{* *} p<0.01,{ }^{* * *} p<0.001$.

\subsection{DSG2 Knockdown Recapitulates Some, but Not All, Effects of DSG2 c.2358 Variant in Control hiPSC-CMs}

To interrogate the role that overall DSG2 expression plays in mediating the biomolecular and electrophysiological changes observed in the DSG2 ${ }^{\text {Mut }}$ hiPSC-CMs, we used small interfering RNA (siRNA) to suppress expression of DSG2 in control cells. After $72 \mathrm{~h}$, siRNA transfection of Ctrl1 hiPSC-CMs substantially reduced DSG2 expression at both the tran- 
scriptional and protein levels, with an $81 \%$ decrease in mRNA abundance (Figure 7A,B) which is a greater reduction than presents in the $D S G 2^{\text {Mut }}$ cells at baseline relative to controls. We did not observe a significant change in the localization of desmoglein-2 in response to DSG2 knockdown (DSG2-KD), nor did we find any effect on the expression or localization of plakoglobin (Figure 7A).
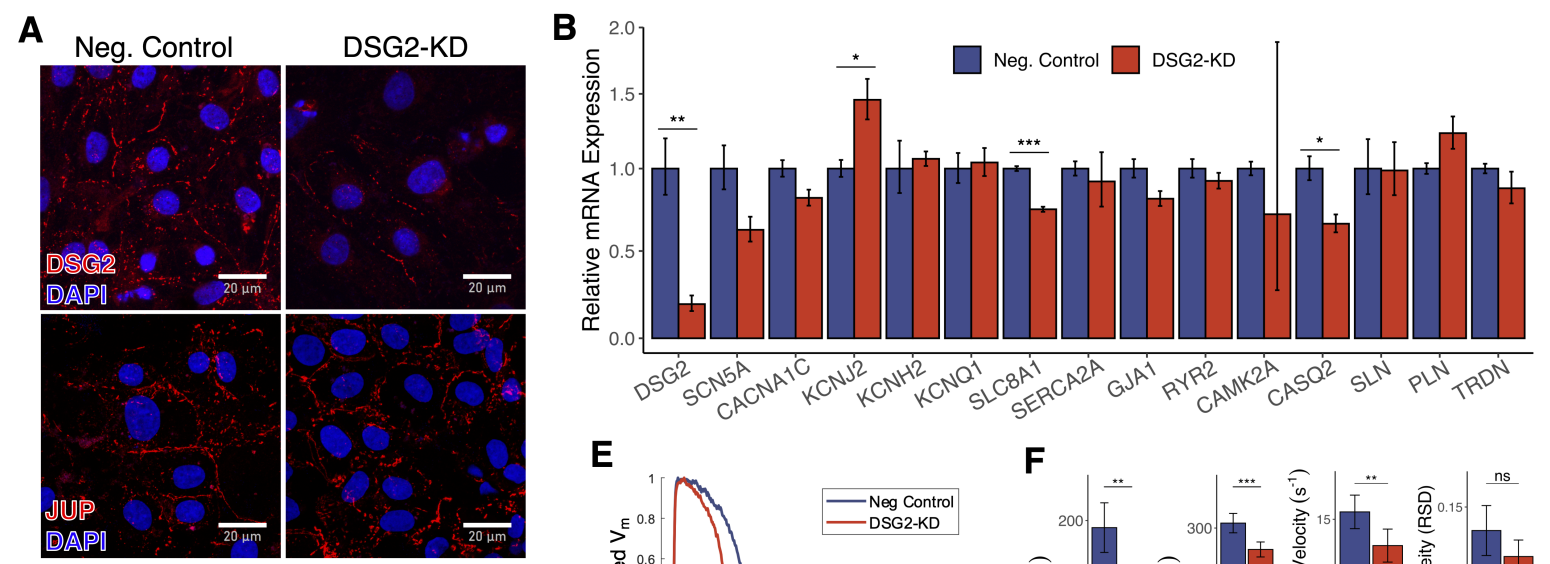
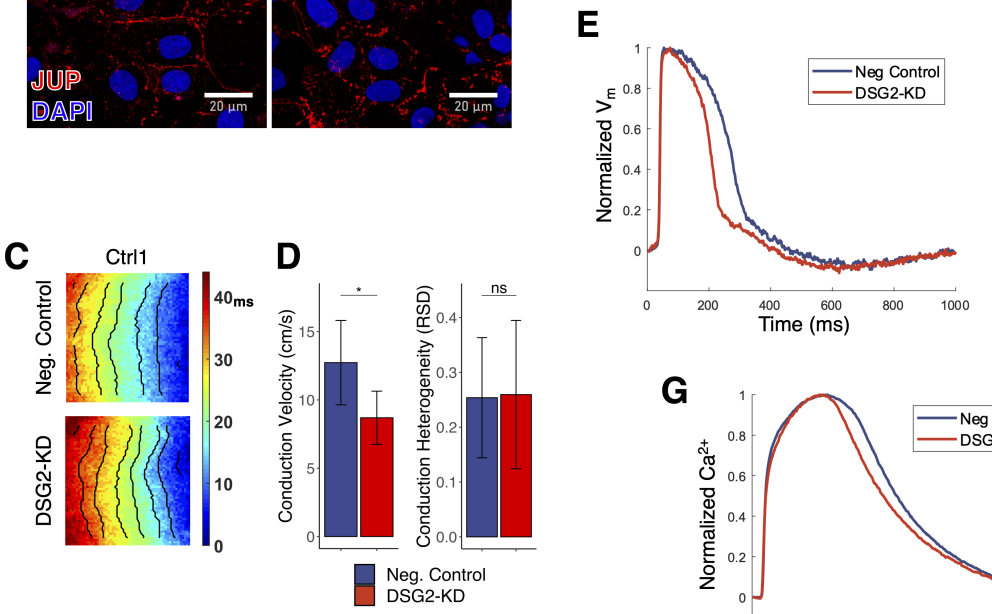
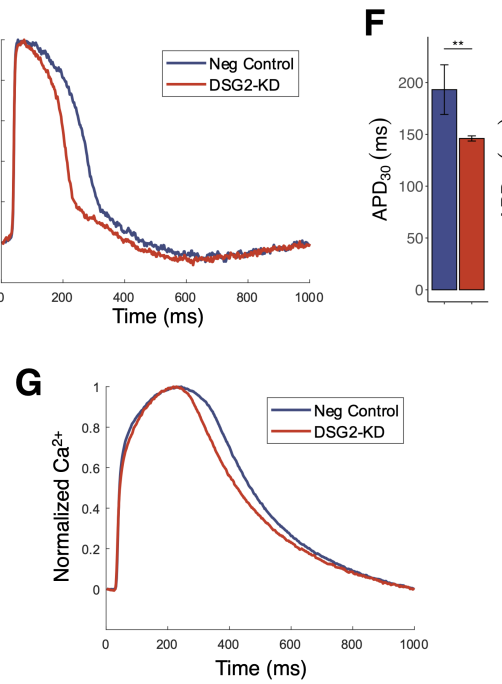

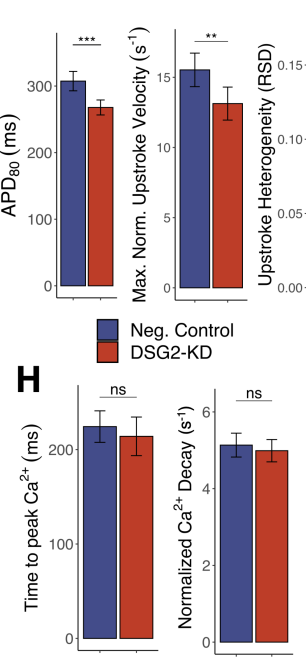

Figure 7. Effects of DSG2 suppression on Ctrl1 hiPSC-CMs. (A) Immunostaining for desmoglein-2 and plakoglobin in Ctrl1 hiPSC-CMs treated with anti-DSG2 and negative control siRNA. White scale bars, $20 \mu \mathrm{m}$. (B) RT-qPCR measurements of key transcripts in Ctrl1 hiPSC-CMs with and without DSG2 knockdown (C) Activation maps with 5 ms isochrones and (D) mean conduction velocities and conduction heterogeneity of Ctrl1 hiPSC-CMs with DSG2 knockdown (E) Representative $\mathrm{V}_{\mathrm{m}}$ traces of Ctrl1 hiPSC-CMs with DSG2 knockdown and (F) mean action potential characteristics. (G) Representative Ca ${ }^{2+}$ traces for Ctrl1 hiPSC-CMs with DSG2 knockdown and $(\mathbf{H})$ mean $\mathrm{Ca}^{2+}$ transient characteristics. Neg. Control $(n=9)$; DSG2-KD $(n=8) .{ }^{*} p<0.05,{ }^{* *} p<0.01,{ }^{* * *} p<0.001, \mathrm{~ns}=$ no significant difference.

DSG2 knockdown resulted in altered abundance of RNAs encoding several ion channels including reduced SLC8A1 ( $\left.\mathrm{I}_{\mathrm{NCX}}\right)$ and increased KCNJ2 $\left(\mathrm{I}_{\mathrm{K} 1}\right)$ (Figure 7B), but notably its effects on SCN5A and KCNQ1 transcript levels, which were altered in DSG2 ${ }^{\mathrm{Mut}} \mathrm{CMs}$ (Figure 3A), were not statistically significant. Expression of calcium handling genes $R Y R 2$, and $S L N$, which were all differentially expressed in $D S G 2^{\text {Mut }} \mathrm{CMs}$, were not significantly affected by DSG2 suppression.

Optical mapping showed that DSG2 knockdown in healthy hiPSC-CM monolayers shortened the action potential duration (Figure 7E,F). At 1000 ms pacing rates, $\mathrm{APD}_{30}$ was reduced by $47 \mathrm{~ms}$ and $\mathrm{APD}_{80}$ was reduced by $40 \mathrm{~ms}$ in Ctrl1 CMs, similar to the differences seen between untreated control and DSG2 ${ }^{\text {Mut }}$ CMs. However, unlike our observation in DSG2 ${ }^{\text {Mut }}$ cells, DSG2-suppressed Ctrl1 cells displayed slower conduction velocities (Figure 7C,D) and slower AP upstroke velocity, without significant effect on the spatial heterogeneity of either parameter (Figure 7F). Interestingly, similar action potential and conduction effects were observed in DSG2 ${ }^{\text {Mut }}$ hiPSCs in response to DSG2 
knockdown (Figure S7). We also found that calcium transients in Crtl1 CMs were not substantively altered by DSG2 knockdown; times-to-peak $\mathrm{Ca}^{2+}$ and $\mathrm{Ca}^{2+}$ decay rates were similar between treated and untreated monolayers (Figure 7G,H).

\section{Discussion}

In this study we established a novel patient-specific hiPSC model of ARVC from an individual with a pathogenic variant in DSG2, the second most common gene associated with ARVC [49]. We performed a broad characterization of cardiomyocytes derived from these hiPSCs, evaluating their biomolecular features as well as their electrophysiological behaviors in monolayer cultures. Our studies confirmed reduced DSG2 expression and disrupted protein localization in $D S G 2^{\text {Mut }} \mathrm{CMs}$, with no apparent changes in expression or localization of other key desmosomal components. We also found that these cells have aberrant electrophysiology and altered $\mathrm{Ca}^{2+}$ handling when compared to wild-type cells, including shortened action potential duration and time-to-peak calcium, as well as altered immune cytokine expression.

The DSG2 c.2358delA variant results in a frameshift and early termination in the intracellular cadherin-like domain of DSG2. It was previously demonstrated that the more distal C-terminal domains are critical in stabilizing desmoglein-2 at the cell membrane, and that deletion of those downstream regions results in increased protein internalization and consequently weakened cell-cell cohesion [50]. Our observations are consistent with those findings: we found desmoglein-2 accumulates intracellularly (primarily perinuclear) in CMs from the mutant line, whereas it was predominantly localized at myocyte-myocyte junctions in controls.

Late-stage ARVC has several well-established pathological markers in human myocardium; however, establishing a definition and criteria constituting a faithful in vitro model of ARVC has proven difficult. Some authors have proposed using reduced plakoglobin expression at cell boundaries as a key component of that definition, since this feature has been shown in both animal [40] and hiPSC [51] models and has even been evaluated as a potential diagnostic marker in humans [52-54]. However, plakoglobin dysregulation has not been observed universally, and notably the extent of dysregulation has been variable among pathogenic variants studied. In our model, plakoglobin expression was not appreciably altered in transcription, protein abundance, or in its localization. Interestingly though, myofibril organization did seem to be affected in DSG2 ${ }^{\text {Mut }}$ hiPSC-CMs, as we found that myofibrillar diameter was significantly reduced in these cells compared to control lines. Studies of myocardial biopsies of ARVC patients have shown altered or degraded myofibrillar structure and some forms of ARVC are associated with myofibrillar myopathy [55,56]. If similar myofibrillar differences are found in other hiPSC models of ARVC, it could offer a novel criterion for defining ARVC phenotype in vitro.

Inflammation is a pathological hallmark of ARVC and occurs during so called "hot phases" of ARVC pathogenesis, further contributing to cardiac remodeling, myocyte cell death and myocardial fibrotic replacement [57]. It is not yet known whether these inflammatory periods are the result of extrinsic factors (e.g., immune cell infiltration) or intrinsic CM dysregulation (e.g., NFkB-mediated cytokine secretion from CMs), but they likely involve both. Specifically, prior reports show infiltration of immune cells in hearts of animal models of ARVC [14,15] and patients with ARVC [58], indicating that extrinsic inflammatory pathways are activated. Yet, our cytokine data in DSG2 ${ }^{\mathrm{Mut}}$ hiPSC-CMs largely corroborates prior reports in PKP2-mutant hiPSC-CMs [15] and clearly demonstrate an intrinsic, NFkB-mediated inflammatory pathway that is dysregulated in hiPSC-CMs, independent of nonmyocyte infiltration. Considering the numerous chemotactic cytokines and inflammation-associated gene transcripts upregulated in purified DSG2 ${ }^{\mathrm{Mut}}$ hiPSC-CM cultures when compared to wild-type cells, this intrinsic pathway may be the driving force for immune cell recruitment to ARVC hearts. Specifically, our RNA-Seq data supports a causative role for canonical NFKB signaling through an IRAK-TAB/TAK-RELA pathway and non-canonical NFKB signaling through a TRAF-IKKa (CHUK) -RELB pathway [14]. 
More broadly, the cytokine profiles in these $D S G 2^{\mathrm{Mut}}$ cells indicates a global upregulation of inflammation machinery and immune cell recruitment. For example, LIF-also known as differentiation stimulating-factor (DSF) - was found to be upregulated in DSG2 ${ }^{\text {Mut }}$ hiPSC-CMs and is a strong inducer of hematopoietic cell differentiation, e.g., myeloid cells into macrophages or pleiotropic cells into T-cells. Both of these terminally differentiated immune cells are routinely found in ARVC hearts [58]. We also found myeloperoxidase and growth regulated oncogene alpha (GRO $\alpha)$ to be upregulated in DSG2 ${ }^{\text {Mut }}$ hiPSC-CMs; both of these cytokines are powerful contributors to cardiac fibrosis via recruitment of neutrophils (immune signaling) together with direct effects on fibroblast proliferation and differentiation [56].

Prior studies of ARVC hiPSC-CMs have reported a range of electrophysiological characteristics. An hiPSC-CM model harboring a compound pathogenic variant in PKP2 showed abnormal sodium channel function as indicated by slowed upstroke speeds and reduced total sodium current [24]. A study of DSG2 p.G638R hiPSC-CMs found similar defects, and another study evaluating hiPSC-CMs derived from a patient with a rare homozygous DSG2 mutation (p.R119X) found very slow conduction velocities compared to an isogenic line with one wildtype DSG2 allele [25,59]. In our model, we did not observe any significant differences between the DSG2-mutant CMs and control CMs in terms of their conduction velocity or relative AP upstroke velocity, though we did find that the DSG2 $2^{\text {Mut }}$ CMs displayed much greater upstroke heterogeneity within each monolayer compared to the controls. Because the cardiomyocyte monolayers used in this study possess electrically connected myocytes and syncytial behavior, our metrics of excitability also reflect sourceload current effects and therefore can differ from single-cell measurements of excitability. Nevertheless, when considered alongside our finding that expression of the cardiac sodium channel (SCN5A) is not reduced in DSG2 ${ }^{\text {Mut }}$ cells, these results are inconsistent with the findings of major intrinsic sodium current dysfunction reported in those prior studies.

In the study of DSG2 p.G638R hiPSCs, the authors also found reduced expression and activity of multiple potassium channels, including $I_{\mathrm{to}}, I_{\mathrm{SK}}$, and $I_{\mathrm{KATP}}$ [25]. Whereas they did not report any significant difference in repolarization dynamics in those cells, we measured substantially shortened action potential durations in our DSG2-mutant CMs as compared to controls. DSG2 ${ }^{\text {Mut }} \mathrm{CMs}$ displayed slightly higher mRNA expression of $K C N Q 1$, the gene encoding the channel responsible for $I_{\mathrm{Ks}}$, which is one of the major repolarizing currents of the action potential. DSG2 ${ }^{\mathrm{Mut}} \mathrm{CMs}$ also had significantly shorter time-to-peak calcium, which may be a consequence of the shortened action potential duration. Interestingly, the ECG of the donor of our DSG2 $2^{\text {Mut }}$ line fulfilled the "major" criteria for ARVC-related repolarization abnormality due to T wave inversions (Figure 1B, Table 1), but had no depolarization abnormality. Given that altered electrophysiology at the cellular level may be reflected in patient ECGs [60], our findings of shortened repolarization times but unchanged conduction velocity and upstroke times are consistent with the ECG findings.

One possible explanation for the differences in action potential characteristics in our model compared to other DSG2-mutant models may be related to alterations in ion channel trafficking, which may be linked to desmoglein-2 and plakophilin-2 trafficking through the formation of macromolecular complexes, such as between Nav1.5 and plakophilin-2 [61], Nav1.5 and desmoglein-2 [11], and Kir2.1 and filamin [62]. The different action potential behavior in our model as compared to other DSG2-mutant models, could be explained by variation in how different mutations affect the ability of DSG2 to form these complexes.

Abnormal $\mathrm{Ca}^{2+}$-handling can promote arrhythmia via a number of different mechanisms and has been implicated in acquired and inherited cardiovascular diseases [63]. Previously reported hiPSC-CM models of ARVC have exhibited accelerated calcium relaxation [23], disrupted calcium homeostasis [17], and increased susceptibility to epinephrineinduced proarrhythmic events (i.e., delayed afterdepolarizations) [25]. Our findings further support the theory that cardiomyocyte calcium regulation is altered in ARVC. In addition to displaying much shorter time-to-peak $\mathrm{Ca}^{2+}$ and slower $\mathrm{Ca}^{2+}$ decay, RT-qPCR exper- 
iments showed overexpression of $R Y R 2$ and reduced expression of $S L N$, which encode the ryanodine receptor and sarcolipin, respectively. Ryanodine receptors regulate calcium release from the sarcoplasmic reticulum (SR), and sarcolipin regulates SR calcium uptake; both are critical for calcium homeostasis [64-66]. High levels of cytosolic $\mathrm{Ca}^{2+}$ have been shown to enhance $I_{\mathrm{Ks}}$ in rabbit cardiomyocytes, which could possibly explain the altered expression of KCNQ1, as well as the shortening of action potential duration observed in DSG2 ${ }^{\text {Mut }}$ hiPSC-CMs [67].

In our model, overall expression of DSG2 was lower in the $D S G 2^{\text {Mut }} \mathrm{CMs}$ than in the controls. To isolate the contributions of a gross difference in DSG2 expression from the potentially dominant negative effects of the c.2358delA variant, we used siRNA targeted against DSG2 mRNA to suppress DSG2 expression without introducing the potential for truncated protein translation. Global suppression of DSG2 in Ctrl1 CMs resulted in shortened action potential durations but had little effect on $\mathrm{Ca}^{2+}$ dynamics. These AP changes are fairly similar to the behavior seen in DSG2 ${ }^{\mathrm{Mut}}$ hiPSC-CMs, but mRNA expression patterns suggest that the AP shortening in the two cases could be the result of different mechanisms. While DSG2 ${ }^{\mathrm{Mut}}$ hiPSC-CMs expressed more of the gene encoding $\mathrm{I}_{\mathrm{Ks}}$ compared to control, DSG2 knockdown did not alter expression in that gene but instead resulted in increased expression of KCNJ2, which encodes $\mathrm{I}_{\mathrm{K} 1}$. RT-qPCR data also offers an explanation for the minimal effects of DSG2 knockdown on calcium dynamics in these cells. Although expression of CASQ2 was reduced by DSG2 knockdown, the other calcium handling genes that were differentially regulated in DSG2 ${ }^{\mathrm{Mut}}$ cells - RYR2 and SLN - were not affected.

While we observed no conduction differences between DSG2 $2^{\text {Mut }}$ and control hiPSCCMs at baseline, we found that DSG2 knockdown caused conduction slowing in control lines. Reduced expression of DSG2 can disrupt connexin-43 localization; indeed, one study showed that mice with a cardiospecific knockout of Dsg2 developed prolonged PR and QRS intervals on ECG recordings, indicative of significant conduction delay [68]. Interestingly, DSG2 knockdown caused similar levels of conduction slowing control and $D S G 2^{\text {Mut }}$ hiPSC-CMs (Figure S7). These results suggest that direct suppression of DSG2 expression is more disruptive of conduction than the DSG2 c.2358delA variant alone.

\section{Study Limitations}

hiPSC-CMs, while a valuable tool, demonstrate a relatively immature phenotype, differing from adult cardiomyocytes phenotypically in several ways. In typical monolayer cultures, they do not assume an elongated morphology or form fully organized intercalated discs, which could affect the level and spatial distribution of desmosomal protein expression. Furthermore, these cells beat spontaneously, and because of their immaturity are more depolarized at rest than adult ventricular cells, which can affect the dynamics of voltage-gated ion channels and alter excitability [69]. On the other hand, hiPSC-CMs cultured in a syncytium such as ours may possess resting transmembrane potentials more similar to adult cardiomyocytes, as compared to single cell preparations [70,71]. We also note that our RT-qPCR studies are limited to three biological replicates for each group. Another limitation is that hiPSC-CMs from the ARVC donor were compared to those from unrelated normal donors, so differences in genetic background are potentially confounding. However, this risk is ameliorated by the use of two independent control lines. Finally, our electrophysiological characterization was limited by the fact the optical mapping system used provided relative, but not absolute, measures of transmembrane potential and $\mathrm{Ca}^{2+}$ concentration.

\section{Conclusions}

In this study we have described an hiPSC-CM model of ARVC derived from a patient with a novel pathogenic mutation in DSG2 and demonstrated a distinct phenotype with altered DSG2 expression, enhanced inflammatory signaling, and electrophysiological aberrations compared with controls. The in vitro repolarization and conduction features 
correlated with the donor's clinical features, and direct suppression of DSG2 in control CMs reproduced many of the mutant line's most prominent phenotypic features. Our findings are generally consistent with observations of ARVC in human studies but differ in several aspects from other published hiPSC-CM models of the disease. This work contributes new evidence that in vitro models of ARVC vary in their behavior significantly, just as ARVC is known to present variably in patients, and underscores the challenge of defining ARVC in vitro in a way that is generalizable across models. Patient-specific hiPSC studies offer the opportunity to systematically investigate this phenotypic variation, which could allow investigators to identify pathophysiological pathways that are common across the disease and to develop broadly useful therapies. Such studies could also provide important insight into attributes of ARVC and other forms of ACM that are specific to different pathogenic variants, aiding the design of more narrowly targeted treatments.

Supplementary Materials: The following are available online at https:/ /www.mdpi.com/article/ 10.3390/jcm10143061/s1, Figure S1: Cardiac MRI for patient JHU013, Figure S2: Cardiac Troponin T expression in hiPSC-CMs, Figure S3: hiPSC-CM expression of pPKA-C and pCaMKII, Figure S4: Lipid production in hiPSC-CMs, Figure S5: Cytokine heatmaps from hiPSC-CM lysates and cell culture supernatants, Figure S6: Conduction velocity aspect ratio in hiPSC-CM monolayers, Figure S7: Optical mapping analysis of siRNA-mediated DSG2 knockdown in DSG2 ${ }^{\text {Mut }}$ hiPSC-CMs, Table S1: RT-qPCR primer sequences, Table S2: siRNA oligo sequences, Table S3: Primary antibodies for immunocytochemistry and Western blot, and Supplementary Methods.

Author Contributions: Conceptualization, R.N.H., A.B., J.L., G.T., L.T.; Methodology, R.N.H., A.B., S.P.C., S.K., K.R.B.; Software, R.N.H., A.B., S.K.; Formal Analysis, R.N.H., S.K., S.P.C.; Investigation, R.N.H., A.B., R.T., D.D., J.L., J.M.-M.; Resources, L.T., G.T., J.E.S.; Data Curation, R.N.H., S.K., C.A.J.; Writing-Original Draft Preparation, R.N.H., S.P.C., D.D.; Writing-Review \& Editing, L.T., G.T., K.R.B., C.A.J., A.B., J.L., J.M.-M.; Visualization, R.N.H., S.P.C., S.K., C.A.J., J.M.-M.; Supervision, L.T., G.T.; Project Administration, R.N.H., L.T., G.T. Funding Acquisition, L.T., G.T., J.E.S. All authors have read and agreed to the published version of the manuscript.

Funding: This work was supported by Maryland Stem Cell Research Fund grant 2016-MSCRF-I-2735 (G.T. and L.T.), MSCRF postdoctoral fellowship (A.B.), the Magic That Matters Fund (G.T.), and NIH grants R01-HL120959 (L.T.), R01-HL152249 (L.T.), R01-HL152249-S2 (R.N.H.), and R01-HL148348 (J.E.S.). The Johns Hopkins ARVD/C Program provided the blood samples used to create the DSG mutant cell line. It is supported by the Leonie-Wild Foundation, the Leyla Erkan Family Fund for ARVD Research, the Francis P. Chiramonte Private Foundation, the Satish, Rupal, and Robin Shah ARVD Fund at Johns Hopkins, the Bogle Foundation, the Healing Hearts Foundation, the Campanella family, the Patrick J. Harrison Family, the Peter French Memorial Foundation, and the Wilmerding Endowments.

Institutional Review Board Statement: The study was approved by and conducted in accordance with the Institutional Review Board of Johns Hopkins Medicine.

Informed Consent Statement: Informed consent was obtained from all subjects involved in the study.

Data Availability Statement: All raw sequencing data as well as count tables and sample information are available on GEO at accession number: GSE176209. Code to replicate results from this manuscript can be found at https://github.com/skannan4/dsg2-mut.

Acknowledgments: We are grateful to the ARVD/C patients and families who have made this work possible. We thank Brittney Murray, Crystal Tichnell, Catherine Pendleton, and Hugh Calkins for their work facilitating the patient donation and maintaining the Johns Hopkins ARVC registry. We also thank Stefan Zimmerman for his assistance in obtaining and interpreting the cardiac magnetic resonance images as well as Bruce Conklin of the University of California, San Francisco for sharing the WTC11 wildtype control hiPSC line.

Conflicts of Interest: The authors declare no conflict of interest.

\section{References}

1. Saguner, A.M.; Brunckhorst, C.; Duru, F. Arrhythmogenic ventricular cardiomyopathy: A paradigm shift from right to biventricular disease. World J. Cardiol. 2014, 6, 154. [CrossRef] 
2. Basso, C.; Corrado, D.; Thiene, G. Cardiovascular causes of sudden death in young individuals including athletes. Cardiol. Rev. 1999, 7, 127-135. [CrossRef]

3. Tabib, A.; Loire, R.; Chalabreysse, L.; Meyronnet, D.; Miras, A.; Malicier, D.; Thivolet, F.; Chevalier, P.; Bouvagnet, P. Circumstances of death and gross and microscopic observations in a series of 200 cases of sudden death associated with arrhythmo-genic right ventricular cardiomyopathy and/or dysplasia. Circulation 2003, 108, 3000-3005. [CrossRef] [PubMed]

4. Basso, C.; Corrado, D.; Marcus, F.I.; Nava, A.; Thiene, G. Arrhythmogenic right ventricular cardiomyopathy. Lancet 2009, 373, 1289-1300. [CrossRef]

5. Peters, S.; Trümmel, M.; Meyners, W. Prevalence of right ventricular dysplasia-cardiomyopathy in a non-referral hospital. Int. J. Cardiol. 2004, 97, 499-501. [CrossRef]

6. Groeneweg, J.A.; Bhonsale, A.; James, C.; Riele, A.S.T.; Dooijes, D.; Tichnell, C.; Murray, B.; Wiesfeld, A.C.P.; Sawant, A.; Kassamali, B.; et al. Clinical presentation, long-term follow-up, and outcomes of 1001 arrhythmogenic right ventricular dysplasia/cardiomyopathy patients and family members. Circ. Cardiovasc. Genet. 2015, 8, 437-446. [CrossRef] [PubMed]

7. Vatta, M.; Marcus, F.; Towbin, J.A. Arrhythmogenic right ventricular cardiomyopathy: A 'final common pathway' that defines clinical phenotype. Eur. Heart J. 2006, 28, 529-530. [CrossRef]

8. Towbin, J.A.; McKenna, W.J.; Abrams, D.; Ackerman, M.J.; Calkins, H.; Darrieux, F.C.; Daubert, J.P.; de Chillou, C.; DePasquale, E.C.; Desai, M.Y.; et al. 2019 HRS expert consensus statement on evaluation, risk stratification, and management of arrhythmogenic cardiomyopathy. Heart Rhythm. 2019, 16, e301-e372. [CrossRef] [PubMed]

9. Hall, C.L.; Sutanto, H.; Dalageorgou, C.; McKenna, W.J.; Syrris, P.; Futema, M. Frequency of genetic variants associated with arrhythmogenic right ventricular cardiomyopathy in the genome aggregation database. Eur. J. Hum. Genet. 2018, 26, 1312-1318. [CrossRef]

10. van Lint, F.H.; Murray, B.; Tichnell, C.; Zwart, R.; Amat, N.; Deprez, R.H.L.; Dittmann, S.; Stallmeyer, B.; Calkins, H.; van der Smagt, J.J.; et al. Arrhythmogenic right ventricular cardiomyopathy-associated desmosomal variants are rarely de novo. Circ. Genom. Precis. Med. 2019, 12, e002467. [CrossRef]

11. Rizzo, S.; Lodder, E.M.; Verkerk, A.; Wolswinkel, R.; Beekman, L.; Pilichou, K.; Basso, C.; Remme, C.A.; Thiene, G.; Bezzina, C.R. Intercalated disc abnormalities, reduced $\mathrm{Na}+$ current density, and conduction slowing in desmoglein-2 mutant mice prior to cardiomyopathic changes. Cardiovasc. Res. 2012, 95, 409-418. [CrossRef]

12. Gomes, J.; Finlay, M.; Ahmed, A.K.; Ciaccio, E.J.; Asimaki, A.; Saffitz, J.E.; Quarta, G.; Nobles, M.; Syrris, P.; Chaubey, S.; et al. Electrophysiological abnormalities precede overt structural changes in arrhythmogenic right ventricular cardiomyopathy due to mutations in desmoplakin-A combined murine and human study. Eur. Heart J. 2012, 33, 1942-1953. [CrossRef]

13. Kaplan, S.R.; Gard, J.J.; Protonotarios, N.; Tsatsopoulou, A.; Spiliopoulou, C.; Anastasakis, A.; Squarcioni, C.P.; McKenna, W.J.; Thiene, G.; Basso, C.; et al. Remodeling of myocyte gap junctions in arrhythmogenic right ventricular cardiomyopathy due to a deletion in plakoglobin (Naxos disease). Heart Rhythm. 2004, 1, 3-11. [CrossRef]

14. Chelko, S.P.; Keceli, G.; Carpi, A.; Doti, N.; Agrimi, J.; Asimaki, A.; Beti, C.B.; Miyamoto, M.; Amat-Codina, N.; Bedja, D.; et al. Exercise triggers CAPN1-mediated AIF truncation, inducing myocyte cell death in arrhythmogenic cardiomyopathy. Sci. Transl. Med. 2021, 13, eabf0891. [CrossRef] [PubMed]

15. Chelko, S.; Asimaki, A.; Lowenthal, J.; Bueno-Beti, C.; Bedja, D.; Scalco, A.; Amat-Alarcon, N.; Andersen, P.; Judge, D.P.; Tung, L.; et al. Therapeutic modulation of the immune response in arrhythmogenic cardiomyopathy. Circulation 2019, 140, 1491-1505. [CrossRef] [PubMed]

16. Cerrone, M.; Montnach, J.; Carolina, V.; Zhao, Y.-T.; Zhang, M.; Agullo-Pascual, E.; Leo-Macias, A.; Alvarado, F.J.; Dolgalev, I.; Karathanos, T.V.; et al. Plakophilin-2 is required for transcription of genes that control calcium cycling and cardiac rhythm. Nat. Commun. 2017, 8, 1-16. [CrossRef]

17. Kim, J.-C.; Pérez-Hernández, M.; Alvarado, F.J.; Maurya, S.; Montnach, J.; Yin, Y.; Zhang, M.; Lin, X.; Vasquez, C.; Heguy, A.; et al. Disruption of $\mathrm{Ca}^{2+}$ i Homeostasis and Connexin 43 Hemichannel Function in the Right Ventricle Precedes Overt Arrhythmogenic Cardiomyopathy in Plakophilin-2-Deficient Mice. Circulation 2019, 140, 1015-1030. [CrossRef] [PubMed]

18. Vischer, A.S.; Connolly, D.J.; Coats, C.J.; Fuentes, V.L.; McKenna, W.J.; Castelletti, S.; Pantazis, A.A. Arrhythmogenic right ventricular cardiomyopathy in Boxer dogs: The diagnosis as a link to the human disease. Acta Myol. 2017, 36, 135-150. [PubMed]

19. Eason, B.D.; Leach, S.B.; Kuroki, K. Arrhythmogenic Right Ventricular Cardiomyopathy in a Weimaraner. Can. Vet. J. 2015, 56, 1035-1039.

20. Boukens, B.J.; Rivaud, M.R.; Rentschler, S.; Coronel, R. Misinterpretation of the mouse ECG: "Musing the waves of mus musculus ". J. Physiol. 2014, 592, 4613-4626. [CrossRef]

21. Ross, S.B.; Fraser, S.; Semsarian, C. Induced pluripotent stem cells in the inherited cardiomyopathies: From disease mechanisms to novel therapies. Trends Cardiovasc. Med. 2016, 26, 663-672. [CrossRef]

22. Blazeski, A.; Lowenthal, J.; Wang, Y.; Teuben, R.; Zhu, R.; Gerecht, S.; Tomaselli, G.; Tung, L. Engineered Heart Slice Model of Arrhythmogenic Cardiomyopathy Using Plakophilin-2 Mutant Myocytes. Tissue Eng. Part. A 2019, 25, 725-735. [CrossRef]

23. Kim, C.; Wong, J.; Wen, J.; Wang, S.; Wang, C.; Spiering, S.; Kan, N.G.; Forcales, S.; Puri, P.L.; Leone, T.C.; et al. Studying arrhythmogenic right ventricular dysplasia with patient-specific iPSCs. Nat. Cell Biol. 2013, 494, 105-110. [CrossRef]

24. Khudiakov, A.; Zaytseva, A.; Perepelina, K.; Smolina, N.; Pervunina, T.; Vasichkina, E.; Karpushev, A.; Tomilin, A.; Malashicheva, A.; Kostareva, A. Sodium current abnormalities and deregulation of Wnt/ $\beta$-catenin signaling in iPSC-derived 
cardiomyocytes generated from patient with arrhythmogenic cardiomyopathy harboring compound genetic variants in plakophilin 2 gene. Biochim. et Biophys. Acta BBA Mol. Basis Dis. 2020, 1866, 165915. [CrossRef] [PubMed]

25. El-Battrawy, I.; Zhao, Z.; Lan, H.; Cyganek, L.; Tombers, C.; Li, X.; Buljubasic, F.; Lang, S.; Tiburcy, M.; Zimmermann, W.-H.; et al. Electrical dysfunctions in human-induced pluripotent stem cell-derived cardiomyocytes from a patient with an arrhythmogenic right ventricular cardiomyopathy. Europace 2018, 20, f46-f56. [CrossRef] [PubMed]

26. Marcus, F.I.; McKenna, W.J.; Sherrill, D.; Basso, C.; Bauce, B.; Bluemke, D.A.; Calkins, H.; Corrado, D.; Cox, M.G.P.J.; Daubert, J.P.; et al. Diagnosis of Arrhytmogenic Right Ventricular Cardiomyopathy/Dysplasia (ARVC/D): Proposed Modification of the Task Force Criteria. Circulation 2010, 121, 1533-1541. [CrossRef] [PubMed]

27. Poon, E.; Luo, X.-L.; Webb, S.; Yan, B.; Zhao, R.; Wu, S.C.M.; Yang, Y.; Zhang, P.; Bai, H.; Shao, J.; et al. The cell surface marker CD36 selectively identifies matured, mitochondria-rich hPSC-cardiomyocytes. Cell Res. 2020, 30, 626-629. [CrossRef] [PubMed]

28. Ong, C.S.; Fukunishi, T.; Zhang, H.; Huang, C.Y.; Nashed, A.; Blazeski, A.; DiSilvestre, D.; Vricella, L.; Conte, J.; Tung, L.; et al. Biomaterial-Free Three-Dimensional Bioprinting of Cardiac Tissue using Human Induced Pluripotent Stem Cell Derived Cardiomyocytes. Sci. Rep. 2017, 7, 1-11. [CrossRef] [PubMed]

29. Miyaoka, Y.; Chan, A.H.; Judge, L.M.; Yoo, J.; Huang, M.; Nguyen, T.D.; Lizarraga, P.P.; So, P.-L.; Conklin, B.R. Isolation of single-base genome-edited human iPS cells without antibiotic selection. Nat. Methods 2014, 11, 291-293. [CrossRef]

30. Burridge, P.W.; Matsa, E.; Shukla, P.; Lin, Z.C.; Churko, J.M.; Ebert, A.D.; Lan, F.; Diecke, S.; Huber, B.; Mordwinkin, N.M.; et al. Chemically Defined and Small Molecule-Based Generation of Human Cardiomyocytes. Nat. Methods 2014, 11, 855-860. [CrossRef] [PubMed]

31. Huang, C.Y.; Maia-Joca, R.P.M.; Ong, C.S.; Wilson, I.; DiSilvestre, D.; Tomaselli, G.F.; Reich, D.H. Enhancement of human iPSC-derived cardiomyocyte maturation by chemical conditioning in a 3D environment. J. Mol. Cell. Cardiol. 2020, 138, 1-11. [CrossRef]

32. Dobin, A.; Davis, C.A.; Schlesinger, F.; Drenkow, J.; Zaleski, C.; Jha, S.; Batut, P.; Chaisson, M.; Gingeras, T.R. STAR: Ultrafast universal RNA-seq aligner. Bioinformatics 2013, 29, 15-21. [CrossRef]

33. Liao, Y.; Smyth, G.K.; Shi, W. featureCounts: An efficient general purpose program for assigning sequence reads to genomic features. Bioinformatics 2014, 30, 923-930. [CrossRef]

34. Liao, Y.; Smyth, G.K.; Shi, W. The Subread aligner: Fast, accurate and scalable read mapping by seed-and-vote. Nucleic Acids Res. 2013, 41, e108. [CrossRef] [PubMed]

35. Love, M.I.; Huber, W.; Anders, S. Moderated estimation of fold change and dispersion for RNA-seq data with DESeq2. Genome Biol. 2014, 15, 550. [CrossRef] [PubMed]

36. Luo, W.; Brouwer, C. Pathview: An R/Bioconductor package for pathway-based data integration and visualization. Bioinformatics 2013, 29, 1830-1831. [CrossRef]

37. Sylvain Le Pepe EasyqpcR: EasyqpcR for Easy Analysis of Real-Time PCR Data. Available online: http://irtomit.labo.univpoitiers.fr / (accessed on 7 January 2021).

38. Richards, S.; Aziz, N.; Bale, S.; Bick, D.; Das, S.; Gastier-Foster, J.; Grody, W.W.; Hegde, M.; Lyon, E.; Spector, E.; et al. Standards and guidelines for the interpretation of sequence variants: A joint consensus recommendation of the American College of Medical Genetics and Genomics and the Association for Molecular Pathology. Genet. Med. 2015, 17, 405-423. [CrossRef] [PubMed]

39. Kelly, M.A.; Caleshu, C.; Morales, A.; Buchan, J.; Wolf, Z.; Harrison, S.M.; Cook, S.; Dillon, M.W.; Garcia, J.; Haverfield, E. Adaptation and validation of the ACMG/AMP variant classification framework for MYH7-associated inherited cardiomyopathies: Recommendations by ClinGen's Inherited Cardiomyopathy Expert Panel. Genet. Med. 2018, 20, 351-359. [CrossRef]

40. Chelko, S.; Asimaki, A.; Andersen, P.; Bedja, D.; Amat-Alarcon, N.; DeMazumder, D.; Jasti, R.; Macrae, C.A.; Leber, R.; Kleber, A.G.; et al. Central role for GSK3 $\beta$ in the pathogenesis of arrhythmogenic cardiomyopathy. JCI Insight 2016, 1, 1. [CrossRef] [PubMed]

41. Asimaki, A.; Kapoor, S.; Plovie, E.; Arndt, A.K.; Adams, E.; Liu, Z.; James, C.; Judge, D.; Calkins, H.; Churko, J.; et al. Identification of a New Modulator of the Intercalated Disc in a Zebrafish Model of Arrhythmogenic Cardiomyopathy. Sci. Transl. Med. 2014, 6, 240ra74. [CrossRef]

42. Toffolo, R.L.; David Ianuzzo, C. Myofibrillar Adaptations during Cardiac Hypertrophy; Kluwer Academic Publishers: New York, NY, USA, 1994; Volume 131.

43. Scheel, P.J.; Murray, B.; Tichnell, C.; James, C.A.; Tandri, H.; Calkins, H.; Chelko, S.P.; Gilotra, N.A. Arrhythmogenic Right Ventricular Cardiomyopathy Presenting as Clinical Myocarditis in Women. Am. J. Cardiol. 2021, 145, 128-134. [CrossRef]

44. Bangert, A.; Andrassy, M.; Müller, A.-M.; Bockstahler, M.; Fischer, A.; Volz, C.H.; Leib, C.; Göser, S.; Korkmaz-Icöz, S.; Zittrich, S.; et al. Critical role of RAGE and HMGB1 in inflammatory heart disease. Proc. Natl. Acad. Sci. USA 2016, 113, E155-E164. [CrossRef]

45. Zhong, Q.; Zhou, K.; Liang, Q.; Lin, S.; Wang, Y.; Xiong, X.; Meng, Z.; Zhao, T.; Zhu, W.; Yang, Y.; et al. Interleukin-23 Secreted by Activated Macrophages Drives $\gamma \delta$ T Cell Production of Interleukin-17 to Aggravate Secondary Injury After Intracerebral Hemorrhage. J. Am. Heart Assoc. 2016, 5. [CrossRef]

46. Ha, H.; Han, D.; Choi, Y. TRAF-Mediated TNFR-Family Signaling. Curr. Protoc. Immunol. 2009, 87, 11-19. [CrossRef]

47. Xie, P. TRAF Molecules in Cell Signaling and in Human Diseases. J. Mol. Signal. 2013, 8, 1-31. [CrossRef] [PubMed]

48. Shi, J.-H.; Sun, S.-C. Tumor Necrosis Factor Receptor-Associated Factor Regulation of Nuclear Factor kB and Mitogen-Activated Protein Kinase Pathways. Front. Immunol. 2018, 9, 1849. [CrossRef] [PubMed] 
49. James, C.A.; Jongbloed, J.D.; Hershberger, R.E.; Morales, A.; Judge, D.P.; Syrris, P.; Pilichou, K.; Domingo, A.M.; Murray, B.; Cadrin-Tourigny, J.; et al. International Evidence Based Reappraisal of Genes Associated With Arrhythmogenic Right Ventricular Cardiomyopathy Using the Clinical Genome Resource Framework. Circ. Genom. Precis. Med. 2021, 14, e003273. [CrossRef] [PubMed]

50. Chen, J.; Nekrasova, O.E.; Patel, D.M.; Klessner, J.L.; Godsel, L.M.; Koetsier, J.L.; Amargo, E.V.; Desai, B.V.; Green, K.J. The C-terminal unique region of desmoglein 2 inhibits its internalization via tail-tail interactions. J. Cell Biol. 2012, 199, 699-711. [CrossRef]

51. Gehmlich, K.; Asimaki, A.; Cahill, T.J.; Ehler, E.; Syrris, P.; Zachara, E.; Re, F.; Avella, A.; Monserrat, L.; Saffitz, J.E.; et al. Novel missense mutations in exon 15 of desmoglein-2: Role of the intracellular cadherin segment in arrhythmogenic right ventricular cardiomyopathy? Heart Rhythm. 2010, 7, 1446-1453. [CrossRef] [PubMed]

52. Asimaki, A.; Tandri, H.; Huang, H.; Halushka, M.K.; Gautam, S.; Basso, C.; Thiene, G.; Tsatsopoulou, A.; Protonotarios, N.; McKenna, W.J.; et al. A New Diagnostic Test for Arrhythmogenic Right Ventricular Cardiomyopathy From the Departments of Pathology. New Engl. J. Med. 2009, 360, 1075-1084. [CrossRef] [PubMed]

53. Munkholm, J.; Christensen, A.H.; Svendsen, J.H.; Andersen, C.B. Usefulness of Immunostaining for Plakoglobin as a Diagnostic Marker of Arrhythmogenic Right Ventricular Cardiomyopathy. Am. J. Cardiol. 2012, 109, 272-275. [CrossRef]

54. Ermakov, S.; Ursell, P.C.; Johnson, C.J.; Meadows, A.; Zhao, S.; Marcus, G.M.; Scheinman, M. Plakoglobin Immunolocalization as a Diagnostic Test for Arrhythmogenic Right Ventricular Cardiomyopathy. Pacing Clin. Electrophysiol. 2014, 37, 1708-1716. [CrossRef]

55. D'Amati, G.; Leone, O.; di Gioia, C.R.T.; Magelli, C.; Arpesella, G.; Grillo, P.; Marino, B.; Fiore, F.; Gallo, P. Arrhythmogenic right ventricular cardiomyopathy: Clinicopathologic correlation based on a revised definition of pathologic patterns. Hum. Pathol. 2001, 32, 1078-1086. [CrossRef]

56. Hedberg, C.; Melberg, A.; Kuhl, A.; Jenne, D.; Oldfors, A. Autosomal dominant myofibrillar myopathy with arrhythmogenic right ventricular cardiomyopathy 7 is caused by a DES mutation. Eur. J. Hum. Genet. 2012, 20, 984-985. [CrossRef]

57. Murray, B. Arrhythmogenic Right Ventricular Dysplasia/Cardiomyopathy (ARVD/C): A Review of Molecular and Clinical Literature. J. Genet. Couns. 2012, 21, 494-504. [CrossRef]

58. Gandjbakhch, E.; Redheuil, A.; Pousset, F.; Charron, P.; Frank, R. Clinical Diagnosis, Imaging, and Genetics of Arrhythmogenic Right Ventricular Cardiomyopathy/Dysplasia. J. Am. Coll. Cardiol. 2018, 72, 784-804. [CrossRef] [PubMed]

59. Shiba, M.; Higo, S.; Kondo, T.; Li, J.; Liu, L.; Ikeda, Y.; Kohama, Y.; Kameda, S.; Tabata, T.; Inoue, H.; et al. Phenotypic recapitulation and correction of desmoglein-2-deficient cardiomyopathy using human-induced pluripotent stem cell-derived cardiomyocytes. Hum. Mol. Genet. 2021. [CrossRef] [PubMed]

60. Klabunde, R.E. Cardiac electrophysiology: Normal and ischemic ionic currents and the ECG. Adv. Physiol. Educ. 2017, 41, 29-37. [CrossRef]

61. Willis, B.C.; Ponce-Balbuena, D.; Jalife, J. Protein assemblies of sodium and inward rectifier potassium channels control cardiac excitability and arrhythmogenesis. Am. J. Physiol. Circ. Physiol. 2015, 308, H1463-H1473. [CrossRef]

62. Sampson, L.J.; Leyland, M.L.; Dart, C. Direct Interaction between the Actin-binding Protein Filamin-A and the Inwardly Rectifying Potassium Channel, Kir2.1. J. Biol. Chem. 2003, 278, 41988-41997. [CrossRef]

63. Sutanto, H.; Lyon, A.; Lumens, J.; Schotten, U.; Dobrev, D.; Heijman, J. Cardiomyocyte calcium handling in health and disease: Insights from in vitro and in silico studies. Prog. Biophys. Mol. Biol. 2020, 157, 54-75. [CrossRef] [PubMed]

64. Bhupathy, P.; Babu, G.J.; Periasamy, M. Sarcolipin and phospholamban as regulators of cardiac sarcoplasmic reticulum Ca ${ }^{2+}$ ATPase. J. Mol. Cell. Cardiol. 2007, 42, 903-911. [CrossRef] [PubMed]

65. Asahi, M.; Otsu, K.; Nakayama, H.; Hikoso, S.; Takeda, T.; Gramolini, A.O.; Trivieri, M.G.; Oudit, G.Y.; Morita, T.; Kusakari, Y.; et al. Cardiac-Specific Overexpression of Sarcolipin Inhibits Sarco(Endo)Plasmic Reticulum Ca 2 ATPase (SERCA2a) Activity and Impairs Cardiac Function in Mice. Proc. Natl. Acad. Sci. USA 2004, 110, 9199-9204. [CrossRef] [PubMed]

66. Zhang, Y.; Matthews, G.D.K.; Lei, M.; Huang, C.L.H. Abnormal Ca ${ }^{2+}$ Homeostasis, Atrial Arrhythmogenesis, and Sinus Node Dysfunction in Murine Hearts Modeling RyR2 Modification. Front. Physiol. 2013, 4, 150. [CrossRef]

67. Bartos, D.C.; Morotti, S.; Ginsburg, K.S.; Grandi, E.; Bers, N.M. Quantitative analysis of the $\mathrm{Ca}^{2+}$-dependent regulation of delayed rectifier K+currentIKsin rabbit ventricular myocytes. J. Physiol. 2017, 595, 2253-2268. [CrossRef]

68. Kant, S.; Holthöfer, B.; Magin, T.M.; Krusche, C.A.; Leube, R.E. Desmoglein 2-Dependent Arrhythmogenic Cardiomyopathy Is Caused by a Loss of Adhesive Function. Circ. Cardiovasc. Genet. 2015, 8, 553-563. [CrossRef]

69. Karbassi, E.; Fenix, A.; Marchiano, S.; Muraoka, N.; Nakamura, K.; Yang, X.; Murry, C.E. Cardiomyocyte maturation: Advances in knowledge and implications for regenerative medicine. Nat. Rev. Cardiol. 2020, 17, 341-359. [CrossRef]

70. van de Sande, D.V.; Kopljar, I.; Maaike, A.; Teisman, A.; Gallacher, D.J.; Bart, L.; Snyders, D.J.; Leybaert, L.; Lu, H.R.; Labro, A.J. The resting membrane potential of hSC-CM in a syncytium is more hyperpolarised than that of isolated cells. Channels 2021, 15, 239-252. [CrossRef]

71. Li, W.; Han, J.L.; Entcheva, E. Syncytium cell growth increases Kir2.1 contribution in human iPSC-cardiomyocytes. Am. J. Physiol. Circ. Physiol. 2020, 319, H1112-H1122. [CrossRef] 